Once again on the supersonic flow separation near a corner

Korolev, G. L. and Gajjar, S. J. B. and Ruban, A. I. 2002

MIMS EPrint: 2006.263

Manchester Institute for Mathematical Sciences

School of Mathematics

The University of Manchester

\footnotetext{
Reports available from: http://eprints.maths.manchester.ac.uk/

And by contacting: The MIMS Secretary

School of Mathematics

The University of Manchester

Manchester, M13 9PL, UK
} 


\title{
Once again on the supersonic flow separation near a corner
}

\author{
By G. L. KOROLEV, J. S. B. GAJJAR AND A. I. RUBAN \\ Department of Mathematics, University of Manchester, Oxford Road, Manchester M13 9PL, UK
}

(Received 7 July 2000 and in revised form 20 April 2001)

Laminar boundary-layer separation in the supersonic flow past a corner point on a rigid body contour, also termed the compression ramp, is considered based on the viscous-inviscid interaction concept. The 'triple-deck model' is used to describe the interaction process. The governing equations of the interaction may be formally derived from the Navier-Stokes equations if the ramp angle $\theta$ is represented as $\theta=$ $\theta_{0} R e^{-1 / 4}$, where $\theta_{0}$ is an order-one quantity and $R e$ is the Reynolds number, assumed large. To solve the interaction problem two numerical methods have been used. The first method employs a finite-difference approximation of the governing equations with respect to both the streamwise and wall-normal coordinates. The resulting algebraic equations are linearized using a Newton-Raphson strategy and then solved with the Thomas-matrix technique. The second method uses finite differences in the streamwise direction in combination with Chebychev collocation in the normal direction and Newton-Raphson linearization.

Our main concern is with the flow behaviour at large values of $\theta_{0}$. The calculations show that as the ramp angle $\theta_{0}$ increases, additional eddies form near the corner point inside the separation region. The behaviour of the solution does not give any indication that there exists a critical value $\theta_{0}^{*}$ of the ramp angle $\theta_{0}$, as suggested by Smith $\&$ Khorrami (1991) who claimed that as $\theta_{0}$ approaches $\theta_{0}^{*}$, a singularity develops near the reattachment point, preventing the continuation of the solution beyond $\theta_{0}^{*}$. Instead we find that the numerical solution agrees with Neiland's (1970) theory of reattachment, which does not involve any restriction upon the ramp angle.

\section{Introduction}

Modern theory of fluid flow separation from a rigid body surface relies, to a significant extent, upon asymptotic analysis of the Navier-Stokes equations at large values of the Reynolds number. Most fruitful in this respect is the method of matched asymptotic expansions. The basic idea of this method belongs to Prandtl, who first used it in his classical study (Prandtl 1904) of fluid flows with small viscosity. In this paper Prandtl was particularly concerned with separation phenomenon and the mathematical means to describe the boundary layer developing along the body surface.

In accordance with Prandtl's theory, a high Reynolds number flow past a rigid body has to be subdivided into two characteristic regions. The main part of the flow may be treated as inviscid. However, for all Reynolds numbers, no matter how large, there always exists a thin region near the wall where the flow is predominantly viscous. Prandtl termed this region the boundary layer, and suggested that it is because of the specific behaviour of this layer that flow separation takes place. Flow development in 
the boundary layer depends on the pressure distribution along the wall. If the pressure gradient is favourable, so that the pressure decreases downstream, then the boundary layer remains well-attached to the wall. However with an adverse pressure gradient, when the pressure starts to rise in the direction of the flow, the boundary layer tends to separate from the body surface. The reason for separation was explained by Prandtl in the following way. Since the velocity in the boundary layer drops towards the wall, the kinetic energy of fluid particles inside the boundary layer appears to be less than that at the outer edge of the boundary layer. In fact the closer a fluid particle is to the wall the smaller appears to be its kinetic energy. This means that whilst the pressure rise in the outer flow may be quite significant, the fluid particles inside the boundary layer may not be able to overcome it. Even a small increase of pressure may cause the fluid particles near the wall to stop and then turn back to form a recirculating flow region characteristic of separated flows.

It might seem surprising that the clear understanding of the physical processes leading to separation could not be converted into a rational mathematical theory for more than half a century. The fact is that the classical boundary-layer theory, which was intended by Prandtl for predicting flow separation, was based on the so-called hierarchical approach where the outer inviscid flow is calculated first ignoring the existence of the boundary layer, and only after that can one turn to the boundary layer analysis. By the late forties it became obvious that such a strategy leads to a mathematical contradiction associated with the so-called Goldstein singularity at the point of separation. The form of this singularity was first described by Landau \& Lifshitz (1944) who demonstrated that the shear stress on the body surface upstream of separation drops as the square root $\sqrt{s}$ of the distance $s$ from the separation, and the velocity component normal to the surface tends to infinity, being inversely proportional to $\sqrt{s}$. This result was later confirmed based on more rigorous mathematical analysis by Goldstein (1948). Goldstein also proved - and this result appeared to be of paramount importance for further development of the boundarylayer separation theory - that the singularity at separation precludes the possibility of continuing the solution beyond the separation point into the region of reverse flow.

Although boundary-layer theory in its classical form was found to be insufficient for describing the separation phenomenon, Prandtl's insight into the physical processes leading to separation and, even more so, the mathematical approach suggested by Prandtl for analysing high Reynolds number flows, laid a foundation for all subsequent studies in the asymptotic theory of separation. In a broader sense, Prandtl's idea of subdividing the entire flow field into a number of regions with distinctively different flow properties proved to be the beginning of one of the most powerful tools in modern asymptotic analysis, the method of matched asymptotic expansions.

Significant progress in the theoretical description of separated flows has been achieved in the last thirty years, and a comprehensive description of the underlying ideas and main results of the theory may be found in the monograph by Sychev et al. (1998). A key element of the separation process, which was not fully appreciated in Prandtl's (1904) classical description, is a mutual interaction between the boundary layer and the external inviscid flow. Because of this interaction, a sharp pressure rise may develop 'spontaneously' at a location on the body surface where in accordance with Prandtl's theory the boundary layer would be well-attached. This pressure rise leads to a rapid deceleration of fluid particles near the wall and formation of the reverse flow downstream of the separation. The interaction precludes development of the Goldstein singularity.

The asymptotic theory of viscous-inviscid interaction, known now as the triple- 
deck theory, was formulated simultaneously by Neiland (1969) and Stewartson \& Williams (1969) for the self-induced separation in supersonic flow and by Stewartson (1969) and Messiter (1970) for incompressible fluid flow near a trailing edge of a flat plate. Later it became clear that the triple-deck interaction region, while being small, plays a key role in many fluid flows. It governs, for instance, upstream influence in the supersonic boundary layer, development of different modes of instabilities, bifurcation of the solution and possible hysteresis in separated flows. As far as separation phenomena are concerned, the theory has been extended to describe boundary-layer separation from a smooth body surface in incompressible fluid flow, supersonic flow separation provoked by a shock wave impinging upon the boundary layer, incipient and large-scale separations at angular points of the body contour both in subsonic and supersonic flows, separation at the trailing edge of a thin aerofoil appearing as a result of increase of the angle of attack or the aerofoil thickness, leading-edge separation, separation of the boundary layer in hypersonic flow on a hot or cold wall, separation provoked by a wall roughness, etc.; see Sychev et al. (1998) and references therein.

However, summarizing the results of a century long effort in this field, we have to admit that despite the significance of the progress made, many aspects of the theory of separated flows remain unresolved. Most notably, the theory remains predominantly restricted to incipient or small-scale separations where the entire recirculating region together with the separation and reattachment points 'fits' into the $O\left(R e^{-3 / 8}\right)$ region of interaction. Even in the studies specifically aimed at describing developed separations (see Neiland 1969; Stewartson \& Williams 1969; Sychev 1972; Ruban 1974), the analysis is confined to the 'local' flow behaviour near the separation point. Meanwhile, the 'global' structure of the flow in the recirculating region remains unresolved.

Analysis of fluid flows with developed separation regions is a long-standing fundamental problem of classical and modern fluid dynamics. It has been the focus of attention of many scientists starting with Helmholtz (1868) and Kirchhoff (1869) who demonstrated that Euler's equations of inviscid fluid motion admit solutions in the class of discontinuous functions which could be used for modelling separated flows. Their approach was later extended and a variety of alternative models of separated flows have been put forward, including those by Squire (1934), Imai (1953, 1957), Batchelor 1956a, b, Roshko (1967), Sychev (1967), Taganov (1970), Sadovskii (1971, 1973), Smith (1985), Peregrine (1985) and Chernyshenko $(1985,1988)$. A critical review of these efforts is given in Chapter 6 of Sychev et al. (1998), leading to the conclusion that all the models suggested so far involve inherent contradictions, except Taganov's (1970) model which is believed to be self-consistent. It was carefully studied by Sadovskii (1973), and then by Chernyshenko (1988) who demonstrated that this model represents the limiting form of the solution of the Navier-Stokes equations as $R e \rightarrow \infty$, representing separated flow past a bluff body. However, even this model is open to criticism. It assumes that as $R e \rightarrow \infty$ the separation zone becomes infinitely large, whereas on its scale the body becomes infinitely small and shrinks to the front stagnation point of the eddy zone. As a consequence it predicts unrealistically low body drag that is independent of the shape of the body. More importantly, Taganov-Chernyshenko's theory cannot be extended to the moderate-scale separations observed, for instance, in the supersonic flow past the compression ramp with $\theta=O(1)$.

To make progress in understanding moderate-scale separation, one can approach the problem from another angle. Considering as an example the compression ramp flow, one can start with the triple-deck description which may be used under the 


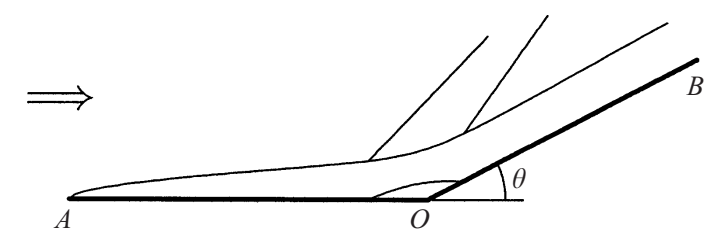

Figure 1. The flow layout. A small separation region is expected to be observed near the corner point $O$, being accompanied by the formation of compression waves in the inviscid flow outside the boundary layer.

assumption that $\theta=R e^{-1 / 4} \theta_{0}$. Then on increasing $\theta_{0}$ the growth of the separation region near the angular point flow may be observed. The appropriate triple-deck formulation for this flow was given by Stewartson (1970) and Neiland (1971b), and early numerical solutions of the triple-deck equations for this flow were produced by Ruban (1978) and Rizzetta et al. (1978). Their calculations revealed that as $\theta_{0}$ becomes large and the size of the separation region increases, the solution near the separation point takes the form characteristic of the separation with a semi-infinite eddy region. Downstream of the separation the 'plateau' zone for pressure is formed. The pressure then rises sharply at reattachment while the skin friction becomes large and negative immediately upstream of the reattachment point.

Later this problem was studied by Smith \& Khorrami (1991). Based on the numerical study of the flow they concluded that a singularity develops in the solution at a finite value $\theta_{0}^{*}$ of the scaled ramp angle $\theta_{0}$. This singularity manifested itself as unlimited increase of the minimal skin friction prior to reattachment as $\theta_{0} \rightarrow \theta_{0}^{*}$, suggesting the breakdown of the solution. If this were to happen, one could argue that the solution would not exist beyond $\theta_{0}^{*}$ which could explain why the theory of moderate-scale separation could not be constructed. However, our calculations do not confirm this result. We will show that while the minimal skin friction does grow very fast for large values of $\theta_{0}$, the solution is found to be in agreement with Neiland's (1970) theory of reattachment, which does not involve any restriction upon the ramp angle.

\section{Formulation of the problem}

Let us consider two-dimensional flow past a compression ramp constructed of two flat plates $A O$ and $O B$, as shown in figure 1. We shall assume that $A O$ is aligned with the oncoming flow, and $O B$ makes an angle $\theta$ with $A O$. Let us denote the velocity, density, viscosity and pressure in the unperturbed free stream by $U_{\infty}, \rho_{\infty}, \mu_{\infty}$ and $p_{\infty}$ respectively; the distance from the leading edge $A$ to the corner point $O$ is $L$.

The Mach number in the free stream is given by

$$
M_{\infty}=\frac{U_{\infty}}{a_{\infty}}, \quad a_{\infty}=\sqrt{\gamma \frac{p_{\infty}}{\rho_{\infty}}},
$$

where $a_{\infty}$ is the speed of sound and $\gamma$ is the specific heats ratio. We shall assume that the oncoming flow is supersonic, i.e. $M_{\infty}$ is an order-one quantity greater than unity. We shall further assume that the compression ramp angle

$$
\theta=R e^{-1 / 4} \theta_{0}
$$




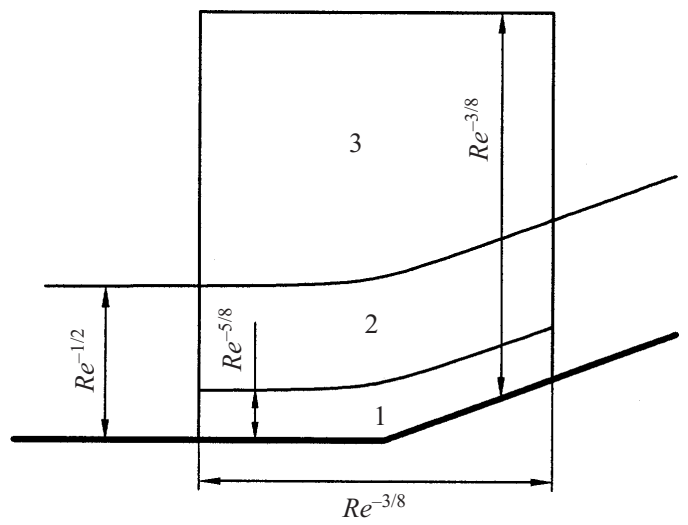

FIGURE 2. Three-tiered structure of the interaction region.

where $\theta_{0}$ is an order-one quantity, and the Reynolds number

$$
R e=\frac{\rho_{\infty} U_{\infty} L}{\mu_{\infty}}
$$

is large.

These are precisely the conditions for the occurrence of incipient separation near the corner point. The separation eddy, at least initially, is entirely embedded in the region of interaction between the boundary layer and inviscid part of the flow, which forms around point $O$. A detailed study of the interaction process for the corner flow was done by Stewartson (1970) and Neiland (1971b). Based on the asymptotic analysis of the Navier-Stokes equations they demonstrated that the region of interaction is $O\left(R e^{-3 / 8}\right)$ and has a three-tiered structure (see figure 2), being composed of the viscous near-wall sublayer (region 1 in figure 2), the main part of the boundary layer (region 2) and an inviscid potential flow region 3 situated outside the boundary layer.

The characteristic thickness of the viscous sublayer is estimated as being an $O\left(R e^{-5 / 8}\right)$ quantity, so that it occupies an $O\left(R e^{-1 / 8}\right)$ portion of the boundary layer and is composed of the stream filaments immediately adjacent to the wall. The flow velocity in this region is $O\left(R e^{-1 / 8}\right)$ relative to the free-stream velocity, and due to the slow motion of gas here the flow exhibits high sensitivity to pressure variations. Even a small pressure rise along the wall may cause significant deceleration of fluid particles there. This leads to thickening of flow filaments, and the streamlines change their shape, being displaced from the wall.

The main part of the boundary layer, the middle tier of the interactive structure, represents a continuation of the conventional boundary layer developing along $A O$ into the interaction region. Its thickness is estimated as $O\left(R e^{-1 / 2}\right)$ and the velocity is an order-one quantity. The flow in this tier is significantly less sensitive to the pressure variations. It does not produce any noticeable contribution to the displacement effect of the boundary layer, which means that all the streamlines in the middle tier are parallel to each other and carry the deformation produced by the displacement effect of the viscous sublayer.

Finally, the upper tier is situated in the potential flow region outside the boundary layer. It serves to 'convert' the perturbations in the form of the streamlines into perturbations of pressure. These are then transmitted through the main part of the boundary layer back to the sublayer.

For separation to take place a certain level of perturbation is necessary. It is 
reached when the scaled ramp angle $\theta_{0}=R e^{1 / 4} \theta$ assumes a critical value. Note that the pressure perturbations in the interaction region are $O\left(R e^{-1 / 4}\right)$. However, due to the slow motion of fluid in the viscous sublayer, a pressure rise of this level is sufficient to cause the fluid particles near the wall become stationary at a certain location on the body surface and then change the direction of their motion, resulting in the formation of a reverse flow region characteristic of separated flows.

To describe the flow in the interaction region we shall start with the viscous sublayer, region 1 in figure 2 . Let $\hat{x}$ and $\hat{y}$ be dimensional coordinates along and normal to the plate $A O$ measured from the corner point $O, \hat{u}$ and $\hat{v}$ be the corresponding velocity components and $\hat{p}$ the pressure. The asymptotic solution of the NavierStokes equations in region 1 is written in the form (see Stewartson 1970; Neiland 1971b)

$$
\left.\begin{array}{l}
\hat{x}=L \operatorname{Re}^{-3 / 8} \frac{\mu_{0}^{-1 / 4} \rho_{0}^{-1 / 2}}{\lambda^{5 / 4} \beta^{3 / 4}} x, \quad \hat{y}=L R e^{-5 / 8} \frac{\mu_{0}^{1 / 4} \rho_{0}^{-1 / 2}}{\lambda^{3 / 4} \beta^{1 / 4}}(y+f(x)), \\
\hat{u}=U_{\infty} \operatorname{Re}^{-1 / 8} \frac{\mu_{0}^{1 / 4} \rho_{0}^{-1 / 2}}{\lambda^{-1 / 4} \beta^{1 / 4}} u, \quad \hat{v}=U_{\infty} \operatorname{Re}^{-3 / 8} \frac{\mu_{0}^{3 / 4} \rho_{0}^{-1 / 2}}{\lambda^{-3 / 4} \beta^{-1 / 4}}\left(v+u \frac{\mathrm{d} f}{\mathrm{~d} x}\right), \\
\hat{p}=p_{\infty}+\rho_{\infty} U_{\infty}^{2} \operatorname{Re}^{-1 / 4} \frac{\mu_{0}^{1 / 2} \rho_{0}^{-1 / 2}}{\lambda^{-1 / 2} \beta^{1 / 2}} p .
\end{array}\right\}
$$

Here $\mu_{0}, \rho_{0}$ and $\lambda$ are suitably non-dimensionalized viscosity, density and skin friction on the wall directly ahead of the triple-deck region, the constant $\beta$ being defined as $\beta=\sqrt{M_{\infty}^{2}-1}$.

Note that the transformations (2.1) serve a threefold purpose. First, they involve the usual rescaling of variables characteristic of the method of matched asymptotic expansions. Second, they include affine transformations which allow us to express the interaction problem in similarity form involving only one controlling parameter

$$
\alpha=\frac{\theta_{0}}{\mu_{0}^{1 / 2} \lambda^{1 / 2} \beta^{1 / 2}} .
$$

Third, the formulae (2.1) also include Prandtl's transposition, which effectively introduces a new curvilinear coordinate system with $x$ measured along the body contour and $y$ in the normal direction.

The body contour is defined in these variables by the function $f(x)$ which for the compression ramp is written as

$$
f(x)=\alpha x H(x), \quad H(x)=\left\{\begin{array}{lll}
0 & \text { if } \quad x<0 \\
1 & \text { if } \quad x \geqslant 0 .
\end{array}\right.
$$

Substitution of (2.1) into the Navier-Stokes equations results in the incompressible form of the Prandtl boundary-layer equations

$$
\begin{gathered}
u \frac{\partial u}{\partial x}+v \frac{\partial u}{\partial y}=-\frac{\mathrm{d} p}{\mathrm{~d} x}+\frac{\partial^{2} u}{\partial y^{2}}, \\
\frac{\partial u}{\partial x}+\frac{\partial v}{\partial y}=0 .
\end{gathered}
$$

They have to be solved with the no-slip condition on the ramp surface

$$
u=v=0 \quad \text { at } \quad y=0,
$$


and the matching conditions with the solutions in the boundary layer upstream of the interaction region

$$
u=y \quad \text { at } \quad x=-\infty
$$

and in the middle tier (region 2)

$$
u \rightarrow y+A(x)+\cdots \quad \text { as } \quad y \rightarrow \infty .
$$

Function $A(x)$ in (2.7) determines the shape of the streamlines in the main part of the boundary layer, and for this reason is termed the displacement function. Using (2.7) in (2.4) it may be easily deduced that at the outer edge of the viscous sublayer $v / u=-\mathrm{d} A / \mathrm{d} x$. As the deformation of the streamlines, produced by the viscous sublayer, remains unchanged across the middle tier (region 2), we can conclude that at the 'bottom' of the upper tier (region 3) the slope of the streamlines is given by $\vartheta=-\mathrm{d} A / \mathrm{d} x+\mathrm{d} f / \mathrm{d} x$. Here, in addition to the displacement effect of the boundary layer the contribution of the body shape is taken into account.

In order to determine the response of the inviscid flow outside the boundary layer to the displacement effect of the boundary layer one has to analyse the Navier-Stokes equations in region 3. This analysis is aimed at deriving the so-called 'interaction law' which relates the induced pressure $p$ to the displacement function $A$. In the case of supersonic flow it is given by Ackeret's formula

$$
p=-\frac{\mathrm{d} A}{\mathrm{~d} x}+\frac{\mathrm{d} f}{\mathrm{~d} x} .
$$

Unlike in Prandtl's classical formulation which presumes that the pressure acting upon the boundary layer is known in advance, the viscous-inviscid interaction allows for the upstream influence through the boundary layer even in well-attached flows, see Lighthill (1953). This means that an additional boundary condition specifying the state of the flow downstream of the interaction region is required. We shall assume that

$$
p=\alpha \quad \text { at } \quad x=\infty .
$$

This condition closes the formulation of the interaction problem (2.3)-(2.9) and makes its solution unique.

\section{Description of numerical methods}

In this section we describe two different numerical approaches used to calculate the solution to (2.3)-(2.9).

\subsection{Method A}

In this method a formulation in terms of the vorticity

$$
\omega=\frac{\partial u}{\partial y}
$$

is adopted. Differentiating (2.3) with respect to $y$ and using (2.4) we arrive at the following equation for $\omega$ :

$$
u \frac{\partial \omega}{\partial x}+v \frac{\partial \omega}{\partial y}=\frac{\partial^{2} \omega}{\partial y^{2}} .
$$


Two boundary conditions for this equation

$$
\omega=1 \text { at } x=-\infty
$$

and

$$
\omega=1 \text { at } y=\infty
$$

immediately follow from (2.6) and (2.7). The third one may be deduced by setting $y=0$ in the momentum equation (2.3) and using the no-slip condition (2.5). This yields

$$
\frac{\partial \omega}{\partial y}=\frac{\mathrm{d} p}{\mathrm{~d} x} \quad \text { at } \quad y=0 .
$$

With known $\omega$ the longitudinal velocity component $u$ may be determined by integrating (3.1) with the first of conditions (2.5) used as an initial condition. We have

$$
u=\int_{0}^{y} \omega \mathrm{d} y_{1} .
$$

Substituting (3.6) into (2.4) and solving for $v$ gives (here the second of conditions (2.5) is used)

$$
v=\int_{0}^{y} y_{1} \frac{\partial \omega}{\partial x} \mathrm{~d} y_{1}-y \int_{0}^{y} \frac{\partial \omega}{\partial x} \mathrm{~d} y_{1} .
$$

Formulae (3.6) and (3.7) allow us to eliminate $u$ and $v$ from (3.2), yielding

$$
\left(\int_{0}^{y} \omega \mathrm{d} y_{1}\right) \frac{\partial \omega}{\partial x}+\left(\int_{0}^{y} y_{1} \frac{\partial \omega}{\partial x} \mathrm{~d} y_{1}-y \int_{0}^{y} \frac{\partial \omega}{\partial x} \mathrm{~d} y_{1}\right) \frac{\partial \omega}{\partial y}=\frac{\partial^{2} \omega}{\partial y^{2}} .
$$

It remains to express the interaction law (2.8) in terms of $\omega$. Substituting (3.6) into (2.7) and differentiating the resulting equation with respect to $x$ we find that the interaction law may be written as

$$
p=-\int_{0}^{\infty} \frac{\partial \omega}{\partial x} \mathrm{~d} y+\frac{\mathrm{d} f}{\mathrm{~d} x} .
$$

For large enough values of $\alpha$ the solution is expected to have a form in which fast variation of the sought functions near the separation point, and even more so near the reattachment point, are accompanied by rather slow variation in the extended eddy region between them. To properly accommodate this type of behaviour we shall use a new deformed longitudinal variable $s$ given by the equation $x=x(s)$ with rescaling coefficient

$$
q=\frac{\mathrm{d} s}{\mathrm{~d} x},
$$

which is to be adjusted based on the results of the calculation.

Introducing the new variable into (3.5), (3.8) and (3.9) we can finally write the interaction problem (3.3)-(3.5), (3.8) and (3.9) in a form convenient for calculations. The problem consists in solving the equation

$$
u \frac{\partial \omega}{\partial s}+\tilde{v} \frac{\partial \omega}{\partial y}=\frac{1}{q} \frac{\partial^{2} \omega}{\partial y^{2}},
$$

where

$$
u=\int_{0}^{y} \omega \mathrm{d} y_{1}, \quad \tilde{v}=\int_{0}^{y} y_{1} \frac{\partial \omega}{\partial s} \mathrm{~d} y_{1}-y \int_{0}^{y} \frac{\partial \omega}{\partial s} \mathrm{~d} y_{1},
$$


subject to the boundary conditions

$$
\begin{gathered}
\omega=1 \quad \text { at } \quad s=-\infty, \\
\omega=1 \quad \text { at } \quad y=\infty, \\
\frac{\partial \omega}{\partial y}=q \frac{\mathrm{d} p}{\mathrm{~d} s} \quad \text { at } \quad y=0,
\end{gathered}
$$

and the interaction law

$$
p=-q \int_{0}^{\infty} \frac{\partial \omega}{\partial s} \mathrm{~d} y+\frac{\mathrm{d} f}{\mathrm{~d} x} .
$$

To construct the numerical solution of the interaction problem (3.10)-(3.15) the following finite-difference technique has been used. We introduce a mesh

$$
\left(y_{k}, s_{j}\right), \quad\left\{\begin{array}{c}
k=1, \ldots, M, \\
j=1, \ldots, N,
\end{array}\right.
$$

and denote the values of $\omega$ at the node points $\left(y_{k}, s_{j}\right)$ by $\omega_{k, j}$; considered together they constitute the grid function $\left\{\omega_{k, j}\right\}$. As pressure $p$ is independent of $y$, it may be represented by the grid function $\left\{p_{j}\right\}$ whose elements are defined as $p_{j}=p\left(s_{j}\right)$.

Choosing the left-hand-side boundary of the computational domain sufficiently far upstream of the corner, we write boundary condition (3.12) in the form

$$
L_{k, 1}=\omega_{k, 1}-1=0, \quad k=1, \ldots, M .
$$

Here and in the following, $L_{k, j}$ denote finite-difference operators acting upon the grid functions $\left\{\omega_{k, j}\right\}$ and $\left\{p_{j}\right\}$. In particular, each operator in (3.16) is written as $L_{k, 1}=\omega_{k, 1}-1$. Setting $L_{k, 1}$ to zero constitutes the corresponding finite-difference equation, the number of which should coincide with the number of elements in $\left\{\omega_{k, j}\right\}$ and $\left\{p_{j}\right\}$.

Boundary condition (3.13) may be represented using this notation as

$$
L_{M, j}=\omega_{M, j}-1=0, \quad j=2, \ldots, N .
$$

We shall now turn to equation (3.10). For the node points on the grid line next to the upstream boundary the Crank-Nicolson approximation for $\partial \omega / \partial s$ has been adopted, leading to the following second-order-accurate finite-difference scheme for (3.10) which is intended to be used for $j=2$ and all $k=2, \ldots, M-1$ :

$$
L_{k, j}=\frac{1}{2}\left(u_{k, j}+u_{k, j-1}\right) \frac{\omega_{k, j}-\omega_{k, j-1}}{s_{j}-s_{j-1}}+\tilde{v}_{k, j-1 / 2} \frac{\lambda_{y} \omega_{k, j}+\lambda_{y} \omega_{k, j-1}}{2}-\frac{\lambda_{y y} \omega_{k, j}+\lambda_{y y} \omega_{k, j-1}}{2 q}=0 .
$$

In order to reduce the number of unknowns and ensure that the operators $L_{k, j}$ in (3.18) act upon $\left\{\omega_{k, j}\right\}$ only, we express $u_{k, j}$ and $\tilde{v}_{k, j}$ using (3.11):

$$
\begin{aligned}
& u_{k, j}=\frac{1}{2}\left[\omega_{1, j}\left(y_{2}-y_{1}\right)\right.\left.+\omega_{k, j}\left(y_{k}-y_{k-1}\right)+\sum_{l=2}^{k-1} \omega_{l, j}\left(y_{l+1}-y_{l-1}\right)\right], \\
& \tilde{v}_{k, j-1 / 2}=\frac{1}{2\left(s_{j-1}-s_{j}\right)}\left[\left(\omega_{1, j}-\omega_{1, j-1}\right)\left(y_{k}-y_{1}\right)\left(y_{2}-y_{1}\right)\right. \\
&\left.+\sum_{l=2}^{k-1}\left(\omega_{l, j}-\omega_{l, j-1}\right)\left(y_{k}-y_{l}\right)\left(y_{l+1}-y_{l-1}\right)\right] .
\end{aligned}
$$


The symbols $\lambda_{y}$ and $\lambda_{y y}$ in (3.18) stand for finite-difference operators which represent the first- and second-order derivatives with respect to $y$. Applied to the vorticity they are written as

$$
\begin{gathered}
\lambda_{y} \omega_{k, j}=\frac{-\xi^{2} \omega_{k-2 \beta, j}+\omega_{k-\beta, j}-\left(1-\xi^{2}\right) \omega_{k, j}}{\left(y_{k-2 \beta}-y_{k}\right)\left(\xi-\xi^{2}\right)}, \\
\lambda_{y y} \omega_{k, j}=2 \frac{v \omega_{k-2 \beta, j}+\delta \omega_{k-\beta, j}+\omega_{k+\beta, j}-(1+\delta+v) \omega_{k, j}}{\left(v+\delta \xi^{2}+\gamma^{2}\right)\left(y_{k-2 \beta}-y_{k}\right)^{2}} .
\end{gathered}
$$

Here

$$
\beta=\left\{\begin{array}{lll}
\operatorname{sign} \tilde{v}_{k, j-1 / 2} & \text { if } & k \neq 2, M-1 \\
-1 & \text { if } & k=2 \\
1 & \text { if } & k=M-1
\end{array}\right.
$$

and

$$
\gamma=\frac{y_{k+\beta}-y_{k}}{y_{k-2 \beta}-y_{k}}, \quad \xi=\frac{y_{k-\beta}-y_{k}}{y_{k-2 \beta}-y_{k}}, \quad \delta=\frac{\gamma-\gamma^{3}}{\xi^{3}-\xi}, \quad v=\frac{\gamma^{3}-\gamma \xi^{2}}{\xi^{2}-1} .
$$

Substitution of expressions (3.19)-(3.22) into (3.18) allows us to calculate the values of $L_{k, j}$ for any distribution of $\left\{\omega_{k, j}\right\}$ in the flow field. The scheme is stable provided that the longitudinal velocity $u$ is positive. This, of course, is valid for the upstream boundary $(j=1)$ and for the grid line next to it $(j=2)$. However, further downstream the longitudinal velocity component $u$ changes its sign as the separation takes place. It then might become positive again if secondary separation occurs inside the primary separation region, which indeed was observed in our calculation for ramp angles larger than $\alpha \simeq 4.6$. The fragmentation of the flow separation is expected to continue as the ramp angle $\alpha$ increases, and to handle these situations one needs to make the scheme stable independently of the sign of $u$. This has been achieved in our study by using in (3.10), instead of the Crank-Nicolson approximation, windward finite-differencing for $\partial \omega / \partial s$ given by

$$
\lambda_{s} \omega_{k, j}=\frac{1}{s_{j}-s_{j-\alpha}}\left[\frac{\omega_{k, j-2 \alpha}}{\mu(\mu-1)}-\frac{\mu}{\mu-1} \omega_{k, j-\alpha}+\frac{1+\mu}{\mu} \omega_{k, j}\right],
$$

where

$$
\alpha=\operatorname{sign} u_{k, j}, \quad \mu=\frac{s_{j}-s_{j-2 \alpha}}{s_{j}-s_{j-\alpha}} .
$$

With (3.23) equation (3.10) is represented by the scheme

$$
L_{k, j}=u_{k, j} \lambda_{s} \omega_{k, j}+\tilde{v}_{k, j} \lambda_{y} \omega_{k, j}-q^{-1} \lambda_{y y} \omega_{k, j}=0,
$$

which has been used in our calculations for $j=3, \ldots, N$ and $k=2, \ldots, M-1$. The longitudinal velocity $u_{k, j}$ in (3.24) is calculated with the help of (3.19), and we again use formulae (3.21) and (3.22) for the derivatives of $\omega$ with respect to $y$ except now parameter $\beta$ is decided based on the sign of $\tilde{v}_{k, j}$, i.e.

$$
\beta=\left\{\begin{array}{lll}
\operatorname{sign} \tilde{v}_{k, j} & \text { if } \quad k \neq 2, M-1 \\
-1 & \text { if } \quad k=2 \\
1 & \text { if } \quad k=M-1
\end{array}\right.
$$

To calculate the lateral velocity component $\tilde{v}_{k, j}$ in (3.24) and (3.25) we use the 
following approximation of $(3.11 b)$ :

$$
\tilde{v}_{k, j}=\frac{1}{2}\left[-y_{k}\left(y_{2}-y_{1}\right) \lambda_{s} \omega_{1, j}+\sum_{l=2}^{k-1} \lambda_{s} \omega_{l, j}\left(y_{l}-y_{k}\right)\left(y_{l+1}-y_{l-1}\right)\right] .
$$

Here, for $\lambda_{s} \omega_{1, j}$ and $\lambda_{s} \omega_{k, j}$ formula (3.23) is used with $\alpha=1$ which corresponds to the upstream-difference representation of $\partial \omega / \partial s$. However, when the secondary separation forms, for the node points inside the secondary separation region we use in (3.26) the 'central-difference' approximation of $\partial \omega / \partial s$ given by

$$
\lambda_{s} \omega_{k, j}=\frac{1}{s_{j}-s_{j+1}}\left[\frac{\omega_{k, j-1}}{\mu(\mu-1)}-\frac{\mu}{\mu-1} \omega_{k, j+1}+\frac{1+\mu}{\mu} \omega_{k, j}\right],
$$

where

$$
\mu=\frac{s_{j}-s_{j-1}}{s_{j}-s_{j+1}} .
$$

Boundary condition (3.14) on the ramp surface is treated similarly to the vorticity equation (3.10). For $j=2$ we use the Crank-Nicolson approximation

$$
L_{1, j}=\frac{\lambda_{y}^{(\beta=-1)} \omega_{1, j}+\lambda_{y}^{(\beta=-1)} \omega_{1, j-1}}{2}-\frac{1}{2} \frac{p_{j}-p_{j-1}}{s_{j}-s_{j-1}}\left(q_{j}+q_{j-1}\right)=0,
$$

where the operator $\lambda_{y}^{(\beta=-1)}$ is given by (3.21) with $\beta=-1$. For the rest of the grid points on the lower boundary of the computational domain, i.e. for $j=3, \ldots, N$, we use upstream differencing for $\mathrm{d} p / \mathrm{d} s$ in (3.14) leading to

$$
L_{1, j}=\lambda_{y}^{(\beta=-1)} \omega_{1, j}-\lambda_{s}^{(\alpha=1)} p_{j} q_{j}=0 .
$$

Here $\lambda_{s}^{(\alpha=1)}$ is given by (3.23) with $\alpha=1$.

Turning next to the interaction law (3.15) one has to keep in mind that the interaction leads to the upstream influence through the boundary layer even when the flow is well-attached to the body surface. This means that finite differencing of equation (3.15) should be in such a way that the solution in the computational domain depends on the state of the flow at the downstream boundary. This is achieved by using downstream differencing for $\partial \omega / \partial s$ on the right-hand side of (3.15). As a result we have the following set of operator equations:

$$
\begin{aligned}
L_{M+1, j}= & \frac{1}{2}\left(p_{j}+p_{j+1}\right)-\frac{q_{j+1}+q_{j}}{2}\left[\frac{1}{2} \frac{\omega_{j+1,1}-\omega_{j, 1}}{s_{j+1}-s_{j}}\left(y_{2}-y_{1}\right)\right. \\
& \left.+\sum_{k=2}^{M-1} \frac{\omega_{k, j+1}-\omega_{k, j}}{s_{j+1}-s_{j}}\left(y_{k+1}-y_{k-1}\right)\right]-\left.\frac{\mathrm{d} f}{\mathrm{~d} x}\right|_{s=s_{j+1 / 2}}=0
\end{aligned}
$$

which have to be used for $j=1, \ldots, N-1$.

Finally, the downstream boundary condition (2.9) gives

$$
L_{M+1, N}=p_{N}-\alpha=0 .
$$

This completes the formulation of the finite-difference equations. We shall now represent them in a vector form. For this purpose we introduce a set of vector operators $\boldsymbol{L}_{j}$, each being composed of the scalar finite-difference operators $L_{k, j}$, $k=1, \ldots, M+1$ acting on the grid line $s=s_{j}$. We also arrange the unknown quantities into vectors $\Omega_{j}$ by combining the elements of the vorticity grid function 
$\omega_{k, j}$ on the $j$ th grid line with pressure $p_{j}$. We have

$$
\boldsymbol{L}_{j}=\left[\begin{array}{c}
L_{1, j} \\
\vdots \\
L_{k, j} \\
\vdots \\
L_{M, j} \\
L_{M+1, j}
\end{array}\right], \quad \Omega_{j}=\left[\begin{array}{c}
\omega_{1, j} \\
\vdots \\
\omega_{k, j} \\
\vdots \\
\omega_{M, j} \\
p_{j}
\end{array}\right], \quad j=1, \ldots, N .
$$

To adjust the rescaling coefficient $q=\mathrm{d} s / \mathrm{d} x$ to the solution of the problem, we introduce a set of controlling parameters $c_{i}, i=1, \ldots, I$ or, equivalently, a vector

$$
\boldsymbol{C}=\left[\begin{array}{c}
c_{1} \\
\vdots \\
c_{I}
\end{array}\right]
$$

Using this notation one can combine (3.16) with the first $(j=1)$ of $(3.29)$ and write them together in a more compact form

$$
\boldsymbol{L}_{1}\left(\Omega_{1}, \Omega_{2}, \boldsymbol{C}\right)=0 .
$$

Similarly, combining the first $(j=2)$ of (3.17) with (3.18), boundary condition (3.27) and the second $(j=2)$ of $(3.29)$ results in

$$
\boldsymbol{L}_{2}\left(\Omega_{1}, \Omega_{2}, \Omega_{3}, \boldsymbol{C}\right)=0 .
$$

The vector equations for the subsequent grid lines

$$
\boldsymbol{L}_{j}\left(\Omega_{j-2}, \Omega_{j-1}, \Omega_{j}, \Omega_{j+1}, \Omega_{j+2}, \boldsymbol{C}\right)=0, \quad j=3, \ldots, N-2
$$

are composed by combining (3.17) with (3.24), (3.28) and (3.29).

The dependence of $\boldsymbol{L}_{j}$ in (3.33) on $\Omega_{j+1}$ and $\Omega_{j+2}$ comes, first, from equation (3.24) where the windward finite-difference operator (3.23) is used for representing derivative $\partial \omega / \partial s$ in equation (3.10). For all the node points with negative $u_{k, j}$ this operator is calculated using $\omega_{k, j+1}$ and $\omega_{k, j+2}$. However, downstream of the reattachment point the longitudinal velocity $u$ is positive except on the wall, which means that $\boldsymbol{L}_{j}$ does not include $\omega_{k, j+1}$ and $\omega_{k, j+2}$. The solution, nevertheless, remains dependent on the downstream flow behaviour due to the interaction law (3.29) which relates $\Omega_{j}$ to $\Omega_{j+1}$. Taking this into account we can write equation (3.33) for $j=N-1$ as

$$
\boldsymbol{L}_{N-1}\left(\Omega_{N-3}, \Omega_{N-2}, \Omega_{N-1}, \Omega_{N}, \boldsymbol{C}\right)=0 .
$$

On the last grid line $(j=N)$ we use the downstream boundary condition (3.30) instead of the interaction law (3.29), and therefore the corresponding operator equation is written as

$$
\boldsymbol{L}_{N}\left(\Omega_{N-2}, \Omega_{N-1}, \Omega_{N}, \boldsymbol{C}\right)=0 .
$$

To solve equations (3.31)-(3.35) Newton-Raphson linearization has been used. With $\hat{\Omega}_{j}, \hat{\boldsymbol{C}}$ denoting an approximation to the solution given as an initial guess or found from the previous iteration, an improved approximation

$$
\Omega_{j}=\hat{\Omega}_{j}+\Delta \Omega_{j}, \quad C=\hat{\boldsymbol{C}}+\Delta C
$$

may be found by substituting (3.36) into (3.31)-(3.35) and making use of the Taylor 
expansions. This results in the following linear equation for the correction vectors $\Delta \Omega_{j}$ and $\Delta C$ :

$$
\begin{gathered}
\frac{\partial \boldsymbol{L}_{1}}{\partial \Omega_{1}} \Delta \Omega_{1}+\frac{\partial \boldsymbol{L}_{1}}{\partial \Omega_{2}} \Delta \Omega_{2}+\frac{\partial \boldsymbol{L}_{1}}{\partial \boldsymbol{C}} \Delta \boldsymbol{C}=-\boldsymbol{L}_{1} \\
\frac{\partial \boldsymbol{L}_{2}}{\partial \Omega_{1}} \Delta \Omega_{1}+\frac{\partial \boldsymbol{L}_{2}}{\partial \Omega_{2}} \Delta \Omega_{2}+\frac{\partial \boldsymbol{L}_{2}}{\partial \Omega_{3}} \Delta \Omega_{3}+\frac{\partial \boldsymbol{L}_{2}}{\partial \boldsymbol{C}} \Delta \boldsymbol{C}=-\boldsymbol{L}_{2} \\
\frac{\partial \boldsymbol{L}_{j}}{\partial \Omega_{j-2}} \Delta \Omega_{j-2}+\frac{\partial \boldsymbol{L}_{j}}{\partial \Omega_{j-1}} \Delta \Omega_{j-1}+\frac{\partial \boldsymbol{L}_{j}}{\partial \Omega_{j}} \Delta \Omega_{j}+\frac{\partial \boldsymbol{L}_{j}}{\partial \Omega_{j+1}} \Delta \Omega_{j+1} \\
+\frac{\partial \boldsymbol{L}_{j}}{\partial \Omega_{j+2}} \Delta \Omega_{j+2}+\frac{\partial \boldsymbol{L}_{j}}{\partial \boldsymbol{C}} \Delta \boldsymbol{C}=-\boldsymbol{L}_{j}, \quad j=3, \ldots, N-2 \\
\frac{\partial \boldsymbol{L}_{N-1}}{\partial \Omega_{N-3}} \Delta \Omega_{N-3}+\frac{\partial \boldsymbol{L}_{N-1}}{\partial \Omega_{N-2}} \Delta \Omega_{N-2}+\frac{\partial \boldsymbol{L}_{N-1}}{\partial \Omega_{N-1}} \Delta \Omega_{N-1}+\frac{\partial \boldsymbol{L}_{N-1}}{\partial \Omega_{N}} \Delta \Omega_{N}+\frac{\partial \boldsymbol{L}_{N-1}}{\partial \boldsymbol{C}} \Delta \boldsymbol{C}=-\boldsymbol{L}_{N-1}, \\
\frac{\partial \boldsymbol{L}_{N}}{\partial \Omega_{N-2}} \Delta \Omega_{N-2}+\frac{\partial \boldsymbol{L}_{N}}{\partial \Omega_{N-1}} \Delta \Omega_{N-1}+\frac{\partial \boldsymbol{L}_{N}}{\partial \Omega_{N}} \Delta \Omega_{N}+\frac{\partial \boldsymbol{L}_{N}}{\partial \boldsymbol{C}} \Delta \boldsymbol{C}=-\boldsymbol{L}_{N}
\end{gathered}
$$

To solve these equations a generalization of the Thomas elimination technique was employed, based on the recurrent use of the formula

$$
\Delta \Omega_{j}=\mathscr{R}_{j} \Delta \Omega_{j+1}+\mathscr{T}_{j} \Delta \Omega_{j+2}+\mathscr{Z}_{j} \Delta \boldsymbol{C}+\boldsymbol{S}_{j}, \quad j=1, \ldots, N-2 .
$$

Here $\mathscr{R}_{j}$ and $\mathscr{T}_{j}$ are $(M+1) \times(M+1)$ matrices, $\mathscr{Z}_{j}$ is an $(M+1) \times I$ matrix and $\boldsymbol{S}_{j}$ an $(M+1)$-component vector. For $j=N-1$ and $j=N$ formula (3.42) reduces to

$$
\left.\begin{array}{l}
\Delta \Omega_{N-1}=\mathscr{R}_{N-1} \Delta \Omega_{N}+\mathscr{Z}_{N-1} \Delta \boldsymbol{C}+\boldsymbol{S}_{N-1}, \\
\Delta \Omega_{N}=\mathscr{Z}_{N} \Delta \boldsymbol{C}+\boldsymbol{S}_{N} .
\end{array}\right\}
$$

Recursive formulae to calculate the Thomas coefficients $\mathscr{R}_{j}, \mathscr{T}_{j}, \mathscr{Z}_{j}$ and $\boldsymbol{S}_{j}$ may be deduced in the usual way by substituting (3.42) and (3.43) into equations (3.37)-(3.41). With known Thomas coefficients formulae (3.42), (3.43) may be rewritten in the form

$$
\Delta \Omega_{j}=\mathscr{F}_{j} \Delta \boldsymbol{C}+\boldsymbol{G}_{j}, \quad j=1, \ldots, N,
$$

where matrices $\mathscr{F}_{j}$ and vectors $\boldsymbol{G}_{j}$ are calculated recursively using

$$
\left.\begin{array}{l}
\mathscr{F}_{j}=\mathscr{Z}_{j}+\mathscr{R}_{j} \mathscr{F}_{j+1}+\mathscr{T}_{j} \mathscr{\mathscr { F }}_{j+2}, \\
\boldsymbol{G}_{j}=\boldsymbol{S}_{j}+\mathscr{R}_{j} \boldsymbol{G}_{j+1}+\mathscr{T}_{j} \boldsymbol{G}_{j+2},
\end{array}\right\} \quad j=1, \ldots, N-2,
$$

and from $(3.43)$

$$
\begin{aligned}
\mathscr{F}_{N} & =\mathscr{Z}_{N}, \quad \mathscr{F}_{N-1}=\mathscr{R}_{N-1} \mathscr{F}_{N}+\mathscr{Z}_{N-1}, \\
\boldsymbol{G}_{N} & =\boldsymbol{S}_{N}, \quad \boldsymbol{G}_{N-1}=\mathscr{R}_{N-1} \boldsymbol{G}_{N}+\boldsymbol{S}_{N-1} .
\end{aligned}
$$

To determine the unknown vector $\boldsymbol{C}$ we need to formulate a set rules that should be used to rescale the longitudinal coordinate. We shall suppose that these rules may be expressed in the form of $I$ scalar equations relating the controlling parameters $c_{1}, \ldots, c_{I}$ with the sought vorticity and pressure grid functions $\left\{\omega_{k, j}\right\},\left\{p_{j}\right\}$. Equivalently we can use one vector equation which we shall write as

$$
\boldsymbol{Q}\left(\Omega_{1}, \ldots, \Omega_{j}, \ldots, \Omega_{N}, \boldsymbol{C}\right)=0 .
$$

Here $\boldsymbol{Q}$ is an $I$-component vector operator. 


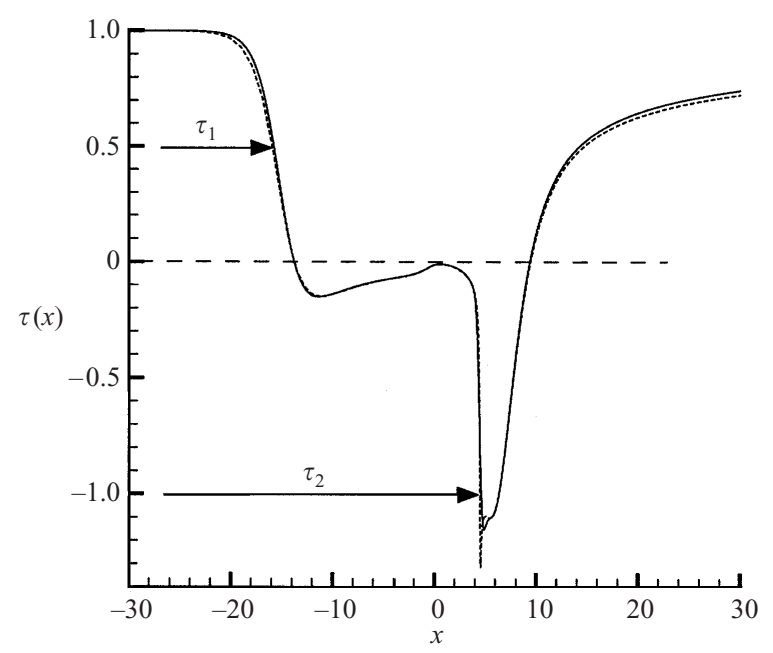

FIGURE 3. Skin friction distribution for $\alpha=4.5$; solid line - method A (mesh $351 \times 101)$; dotted line - method B $($ mesh $1400 \times 71)$.

Equation (3.45) may be linearized in a usual way, leading to

$$
\sum_{j=1}^{N} \frac{\partial \boldsymbol{Q}}{\partial \Omega_{j}} \Delta \Omega_{j}+\frac{\partial \boldsymbol{Q}}{\partial \boldsymbol{C}} \Delta \boldsymbol{C}+\boldsymbol{Q}=0
$$

Combining (3.46) with (3.44) we obtain

$$
\left(\sum_{j=1}^{N} \frac{\partial \boldsymbol{Q}}{\partial \Omega_{j}} \mathscr{F}_{j}+\frac{\partial \boldsymbol{Q}}{\partial \boldsymbol{C}}\right) \Delta \boldsymbol{C}+\sum_{j=1}^{N} \frac{\partial \boldsymbol{Q}}{\partial \Omega_{j}} \boldsymbol{G}_{j}+\boldsymbol{Q}=0 .
$$

This equation should be solved for $\Delta C$ and then the correction vectors $\Delta \Omega_{j}$ may be calculated by making use of formula (3.44). The solution is then updated using (3.36), and the iterations are repeated until the convergence is reached.

The numerical procedure described above was applied to the compression ramp flow calculations in the following way. We started with a relatively small value of the compression ramp angle, namely $\alpha=1.0$, using the unperturbed flow field $\omega_{k, j}=1, p_{j}=0$ for initializing the unknown vectors $\Omega_{j}$. Angle $\alpha$ was then increased progressively, and for each new $\alpha$ the converged solution corresponding to a smaller $\alpha$ was used as an initial guess to start the iterations. Typically seven or eight iterations were needed for convergence to be reached.

For angles up to $\alpha=4.5$ no automatic adjustment of the grid was found necessary, and $s$ was simply set to coincide with $x$. Most of the calculations were performed on two grids, $701 \times 101$ and $351 \times 151$, both being non-uniform. In the $y$-direction the grid step was chosen to grow with the distance from the wall according to a geometric progression. Near the wall it was as small as $y_{2}-y_{1}=0.1$. At the same time the upper boundary of the computational domain was moved out significantly further than in previous studies of the flow. In our calculations it was normally placed at $y_{M}=120$. In the longitudinal direction the grid points were concentrated in a region of large gradients of the sought functions observed just upstream of the reattachment point. The results of the calculations for $\alpha=4.5$ are shown in figure 3 in the form of the 
skin friction $\tau$ plotted against $x$; the skin friction is defined as

$$
\tau=\left.\frac{\partial u}{\partial y}\right|_{y=0} .
$$

We see that a region around $x=4.8$ requires special attention. In this region there were fifty uniformly spaced grid points with the step as small as $s_{j+1}-s_{j}=0.005$. Both upstream and downstream of this region the step size was increased monotonically according to geometric progression. The entire computational domain covered a region stretching from $s_{1}=-30$ to $s_{N}=70$.

Initial attempts to extend the calculations beyond $\alpha=4.5$ showed significant deterioration of the convergence properties of the Newtonian iteration used in our scheme. To enable the scheme to converge we had to decrease the increment of the angle $\alpha$ to $\Delta \alpha=0.005$. The situation was cured by introducing an adaptive grid as follows. Based on the results of calculations for $\alpha=4.5$ two points were identified as 'centres' of large gradient regions. The first one is situated slightly upstream of the separation point, and the other slightly upstream of the minimal skin friction point. They are shown in figure 3 by the arrows. Denoting the $s$-coordinates of these points by $s_{1}$ and $s_{2}$, and the corresponding values of index $j$ by $j_{1}$ and $j_{2}$, we chose the rescaling function to be

$$
x=\left\{\begin{array}{lll}
s+c_{1} \Phi\left(s_{1}\right)+c_{2} & \text { if } & s<s_{1}, \\
s+c_{1} \Phi(s)+c_{2} & \text { if } & s_{1} \geqslant s \geqslant s_{2}, \\
s+c_{2} & \text { if } & s>s_{2},
\end{array}\right.
$$

where

$$
\Phi(s)=\int_{s_{2}}^{s}\left(t-s_{1}\right)^{2}\left(t-s_{2}\right)^{2} \mathrm{~d} t .
$$

The two controlling parameters $c_{1}$ and $c_{2}$ in (3.47) require vector equation (3.45) to be composed of two scalar equations, written as

$$
\begin{aligned}
& Q_{1}=\omega_{1, j_{1}}-\tau_{1}=0, \\
& Q_{2}=\omega_{1, j_{2}}-\tau_{2}=0,
\end{aligned}
$$

which means that for $\alpha>4.5$ points $s=s_{1}$ and $s=s_{2}$ were attributed to the same values of the skin friction as given by the solution for $\alpha=4.5$.

After this modification the convergence of the iteration procedure was fully restored and we could progress to larger values of $\alpha$ with increments $\Delta \alpha=0.1$.

\subsection{Method B}

In the second method equations (2.2) are first written in terms of the stream function $\psi$ where $u=\partial \psi / \partial y, v=-\partial \psi / \partial x$. This method makes use of finite differences in $x$ combined with Chebychev collocation in $y$. In the $y$-direction the domain $0<y<y_{\infty}$ is linearly mapped into $-1<z<1$ and at each $x$-station Chebychev collocation is used to enforce the equations and boundary conditions. So, defining $\psi_{k, j}=\psi\left(x_{j}, y\left(z_{k}\right)\right)$, where $z_{k}=-\cos \left(k \pi / N_{B}\right),\left(0 \leqslant k \leqslant N_{B}\right)$ are the collocation points, the derivative values $\left(\partial^{r} \psi / \partial y^{r}\right)\left(x_{j}, y\left(z_{k}\right)\right)=\mathrm{D}^{r} \psi_{j, k}$ are computed via

$$
\frac{\partial^{r} \psi_{j}}{\partial y^{r}}=\mathbf{D}^{r} \psi_{j}
$$

where $\psi_{j}=\left(\psi_{j, 0}, \psi_{j, 1}, \ldots, \psi_{j, N_{B}}\right)^{T}$ and $\mathbf{D}$ is the Chebychev differentiation matrix operator, see Canuto et al. (1988). 
In the $x$-direction a non-uniform grid is used with grid spacing $h_{j}=x_{j}-x_{j-1}(1 \leqslant$ $j \leqslant M_{B}$ ). The $x$-derivatives occurring in (2.2) are approximated using second-order finite differences as follows. First, in regions of unseparated flow, a three-point backward difference approximation for the $u \partial u / \partial x$ term is used. In regions of separated flow regions either a three-point forward difference operator, or a combination of forward and backward differences are used, dependent on the magnitude of $|u|$. Thus in general this term is approximated as

$$
\begin{aligned}
\frac{\partial \psi}{\partial y} \frac{\partial^{2} \psi}{\partial y \partial x}\left(x_{j}, y\left(z_{k}\right)\right)=(\mathrm{D} \psi)_{j, k}\left[\alpha_{j, k}(\mathrm{D} \psi)_{k, j-2}+\beta_{j, k}(\mathrm{D} \psi)_{k, j-1}\right. \\
\left.+\gamma_{j, k}(\mathrm{D} \psi)_{k, j}+\delta_{j, k}(\mathrm{D} \psi)_{k, j+1}+\sigma_{j, k}(\mathrm{D} \psi)_{k, j+2}\right] .
\end{aligned}
$$

Here

$$
\begin{gathered}
\alpha_{j, k}\left(e_{j, k}\right)=\frac{e_{j, k} h_{j}}{\left(h_{j}+h_{j-1}\right) h_{j-1}}, \quad \beta_{j, k}\left(e_{j, k}\right)=-\frac{e_{j, k}\left(h_{j}+h_{j-1}\right)}{h_{j} h_{j-1}}, \\
\gamma_{j, k}\left(e_{j, k}\right)=\frac{e_{j, k}\left(2 h_{j} h_{j-1}+h_{j-1}^{2}\right)}{h_{j} h_{j-1}\left(h_{j}+h_{j-1}\right)}-\frac{\left(1-e_{j, k}\right)\left(2 h_{j+1} h_{j+2}+h_{j+2}^{2}\right)}{h_{j+1} h_{j+2}\left(h_{j+1}+h_{j+2}\right)}, \\
\delta_{j, k}\left(e_{j, k}\right)=\frac{\left(1-e_{j, k}\right)\left(h_{j+1}+h_{j+2}\right)}{h_{j+2} h_{j+1}}, \quad \sigma_{j, k}\left(e_{j, k}\right)=-\frac{\left(1-e_{j, k}\right) h_{j+1}}{\left(h_{j+2}+h_{j+1}\right) h_{j+2}},
\end{gathered}
$$

and $e_{j, k}$ is a switching function defined by

$$
e_{j, k}=\left\{\begin{array}{lll}
1 & \text { if } \quad \mathrm{D} \psi_{j, k}>\varepsilon \\
0 & \text { if } \quad \mathrm{D} \psi_{j, k}<-\varepsilon,
\end{array}\right.
$$

with $e_{j, k}$ varying linearly from 0 to 1 when $-\varepsilon<\mathrm{D} \psi_{j, k}<\varepsilon$.

A number of different approaches were used for approximating the $v \partial u / \partial y$ term. For the cases when secondary separation was not present a three-point backward second-order difference approximation was used. For the values of the angle when secondary separation arose, a second-order central difference approximation was used to approximate $v$ in the regions of secondary separation, and a three-point second-order backward difference was used elsewhere. Thus the term $v \partial u / \partial y$ was approximated by

$$
\begin{aligned}
\frac{\partial \psi}{\partial x} \frac{\partial^{2} \psi}{\partial y^{2}}\left(x_{j}, y\left(z_{k}\right)\right)=\left(\mathrm{D}^{2} \psi\right)_{j, k}\left[\bar{\alpha}_{j, k}(\psi)_{k, j-2}\right. & +\bar{\beta}_{j, k}(\psi)_{k, j-1} \\
& \left.+\bar{\gamma}_{j, k}(\psi)_{k, j}+\bar{\delta}_{j, k}(\psi)_{k, j+1}+\bar{\sigma}_{j, k}(\psi)_{k, j+2}\right],
\end{aligned}
$$

where

and

$$
\left.\begin{array}{l}
\bar{\alpha}_{j, k}=\bar{\sigma}_{j, k}=0, \quad \bar{\beta}_{j, k}=-\frac{h_{j+1}}{h_{j}\left(h_{j}+h_{j+1}\right)} \\
\bar{\gamma}_{j, k}=\frac{h_{j+1}-h_{j}}{h_{j} h_{j+1}}, \quad \bar{\delta}_{j, k}=\frac{h_{j}}{h_{j+1}\left(h_{j+1}+h_{j}\right)}
\end{array}\right\} \quad \text { for } \quad j_{b} \leqslant j \leqslant j_{e}
$$

$$
\left.\begin{array}{l}
\bar{\alpha}_{j, k}=\alpha_{j, k}(1), \quad \bar{\beta}_{j, k}=\beta_{j, k}(1) \bar{\gamma}_{j, k}=\gamma_{j, k}(1) \\
\bar{\delta}_{j, k}=\delta_{j, k}(1), \quad \bar{\sigma}_{j, k}=\sigma_{j, k}(1)
\end{array}\right\} \quad \text { for } \quad j<j_{b} \quad \text { or } \quad j>j_{e} .
$$

The values $j=j_{s}$ and $j=j_{e}$, denote the $x$-locations of the start and end of the region of secondary separation.

Next, introducing $\bar{A}_{j}=A\left(x_{j}\right)-\alpha f_{0}\left(x_{j}\right)$, the nonlinear system of equations arising 
from the discretization takes the form

$$
\begin{aligned}
(\mathrm{D} \psi)_{j, k}\left[\alpha_{j, k}(\mathrm{D} \psi)_{k, j-2}+\beta_{j, k}(\mathrm{D} \psi)_{k, j-1}+\gamma_{j, k}(\mathrm{D} \psi)_{k, j}+\delta_{j, k}(\mathrm{D} \psi)_{k, j+1}+\sigma_{j, k}(\mathrm{D} \psi)_{k, j+2}\right] & \\
& -\left(\mathrm{D}^{2} \psi\right)_{j, k}\left[\bar{\alpha}_{j, k}(\psi)_{k, j-2}+\bar{\beta}_{j, k}(\psi)_{k, j-1}+\bar{\gamma}_{j, k}(\psi)_{k, j}+\bar{\delta}_{j, k}(\psi)_{k, j+1}+\bar{\sigma}_{j, k}(\psi)_{k, j+2}\right] \\
= & \frac{\bar{A}_{j-1}}{\left(h_{j}+h_{j+1}\right)}-\frac{\bar{A}_{j}}{h_{j+1}}+\frac{h_{j} \bar{A}_{j+1}}{h_{j+1}\left(h_{j}+h_{j+1}\right)}+h_{j}\left(\mathrm{D}^{3} \psi\right)_{j, k} .
\end{aligned}
$$

Equations (3.48) were enforced at the collocation points $z_{k}$ for $1<k<N_{B}-1$. The boundary conditions

$$
\psi_{j, 0}=0, \quad(D \psi)_{j, 0}=0, \quad(D \psi)_{j, N_{B}}=y_{\infty}+A\left(x_{j}\right), \quad\left(D^{2} \psi\right)_{j, N_{B}}=1,
$$

were also used which, together with (3.48), give $\left(N_{B}+2\right)$ equations for the $\left(N_{B}+2\right)$ unknowns $\psi_{j}$ and $A_{j}$ at each $x$-station for $1<j \leqslant M_{B}$. For the first two locations $j=0,1$ the undisturbed solution was used, and in all the computations the value of $x$ corresponding to $j=M_{B}$ was in the unseparated flow region.

The nonlinear system of equations was solved by first introducing a NewtonRaphson linearization with $\psi_{j, k}=\hat{\psi}_{j, k}+\Delta \psi_{j, k}, \bar{A}_{j}=\hat{A}_{j}+\Delta \bar{A}_{j}$, and solving a linear system for the corrections $\Delta \psi_{j, k}, \Delta \bar{A}_{j}$. The hatted quantities represent an approximate solution. The form of (3.48) leads to a block pentadiagonal system for the corrections which was solved using a direct method. Typically only a few Newton iterations were required to obtain the complete solution. For the smaller ramp angles the solution was obtained from an arbitrary initial guess, but for the larger angles a solution for a smaller angle was used to start the computation.

The computations were carried out on uniform as well as non-uniform grids. The non-uniform grid was constructed such that it was divided into three sub-grids in the intervals $\left(-x_{0},-x_{1}\right),\left(-x_{1}, x_{2}\right)$, and $\left(x_{2}, x_{3}\right)$. The values of $x_{0}$ and $x_{3}$ were taken to be large; $x_{1}, x_{2}$ were chosen so as to encompass the separated flow region and in the $\left(-x_{1}, x_{2}\right)$ region the grid was uniform. For $x<-x_{1}$ and $x>x_{2}$, the grid was constructed such that successive intervals were in a geometric progression. Also the bulk of the streamwise points $M_{b}$ were chosen to lie in $\left(-x_{1}, x_{2}\right)$. For the uniform grid computations (say between $\left(-x_{0}, x_{3}\right)$ ) the typical values used were $x_{0}=60, x_{3}=60$. For the non-uniform grid computations, typically 1001 points were used with 801 points concentrated in the separated flow region.

Grid size checks were performed for various values of $M_{b}$, the number of streamwise points, and $N_{b}$, the number of collocation points. It was found that 64 points were sufficient to resolve the flow features in the $y$-direction for angles up to $\alpha=5$. The lack of resolution in the wall-normal direction resulted in oscillations near the point of secondary separation. The value of $y_{\infty}$ was varied and it was found that $y_{\infty}$ had to be increased for increasing values of $\alpha$, and for all of the results shown here the value $y_{\infty}=50$ was used.

\section{Calculation results}

The computations of the compression ramp flow have been performed in this study for a slightly smoothed body shape

$$
f(x)=\frac{\alpha}{2}\left(x+\sqrt{x^{2}+r^{2}}\right)
$$

which was used in the interaction law (2.8) instead of the sharp corner (2.2). This modification does not produce any noticeable influence on the calculation results, 

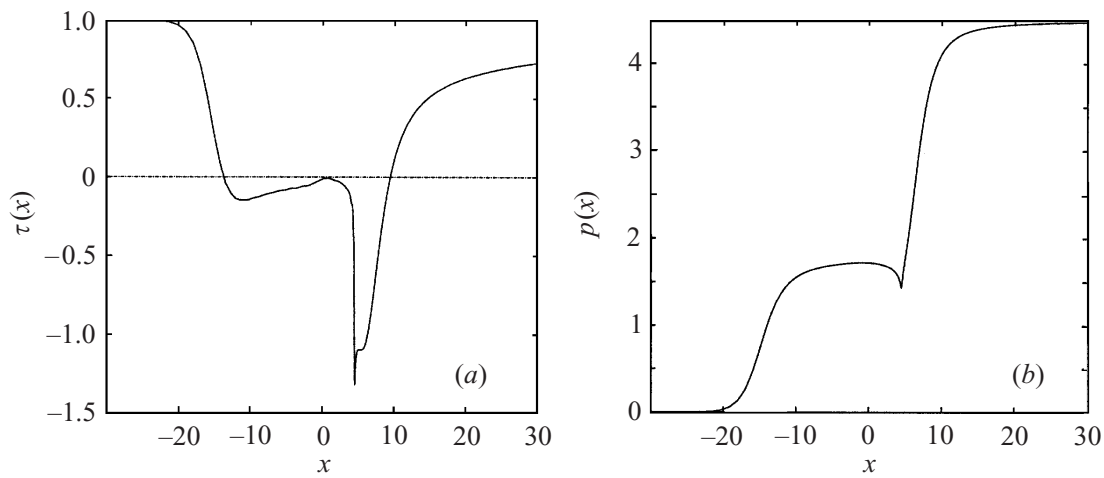

FIGURE 4. Comparison of $(a)$ the skin friction and $(b)$ pressure distributions for the sharp and rounded corners for the ramp angle $\alpha=4.5$. Calculations were performed by method B (mesh $1001 \times 48$ ); solid line - sharp corner; dashed line - rounded corner.
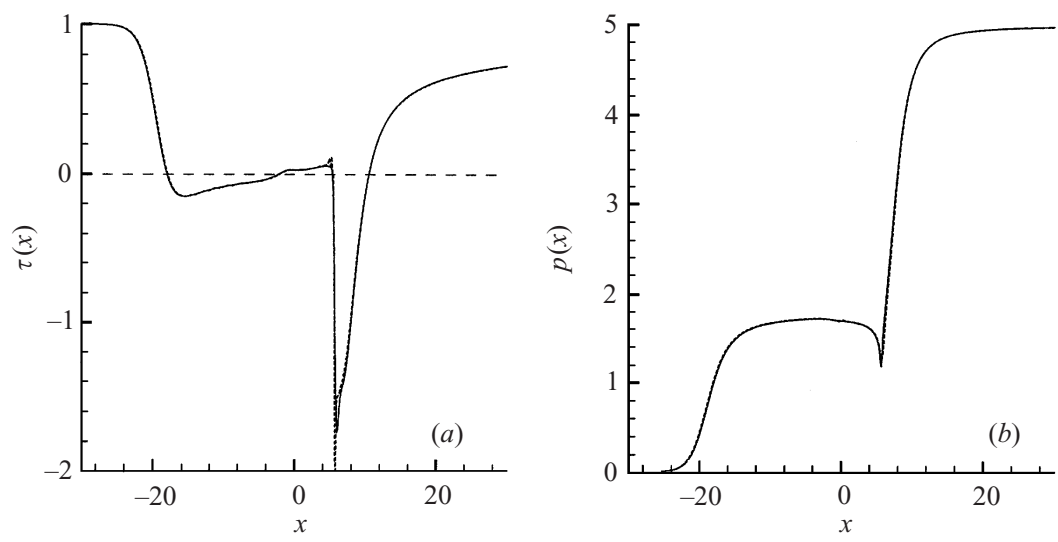

FIGURE 5. Calculations of $(a)$ skin friction and $(b)$ pressure distribution for $\alpha=5$; solid line - method A $($ mesh $1001 \times 201)$; dotted line - method B $($ mesh $1400 \times 71)$.

while it makes the task of numerical modelling of the flow easier. In particular, it avoids special treatment of the corner region where otherwise a concentration of the mesh points would be needed. Figure 4 displays the distributions of the skin friction and pressure along the body surface calculated by method B for the sharp corner with $\alpha=4.5$ and for the corresponding smoothed body. As can be seen the results are graphically indistiguishable. The agreement becomes even better for larger values of $\alpha$ when the corner is deeply embedded in the region of slowly moving reversed flow. In these and all subsequent calculations the smoothing parameter was taken to be $r=0.5$.

For ramp angles up to $\alpha=4.5$ the results produced by methods $\mathrm{A}$ and $\mathrm{B}$ are in excellent agreement with each other (see figure 3). They also agree very well with the previous calculations of Ruban (1978), Rizzetta, Burggraf \& Jenson (1978) and Smith \& Khorrami (1991). However, for larger $\alpha$ important new features in the flow development have been observed, as may be seen using, for example, the results for $\alpha=5$ shown in figure 5. Far upstream of the corner point the flow is unperturbed. The skin friction $\tau=1$, as is required by the boundary condition (3.3), and the pressure $p=0$. Then the pressure starts to rise causing flow deceleration in the boundary layer. The skin friction decreases, crossing zero at the separation point. It 

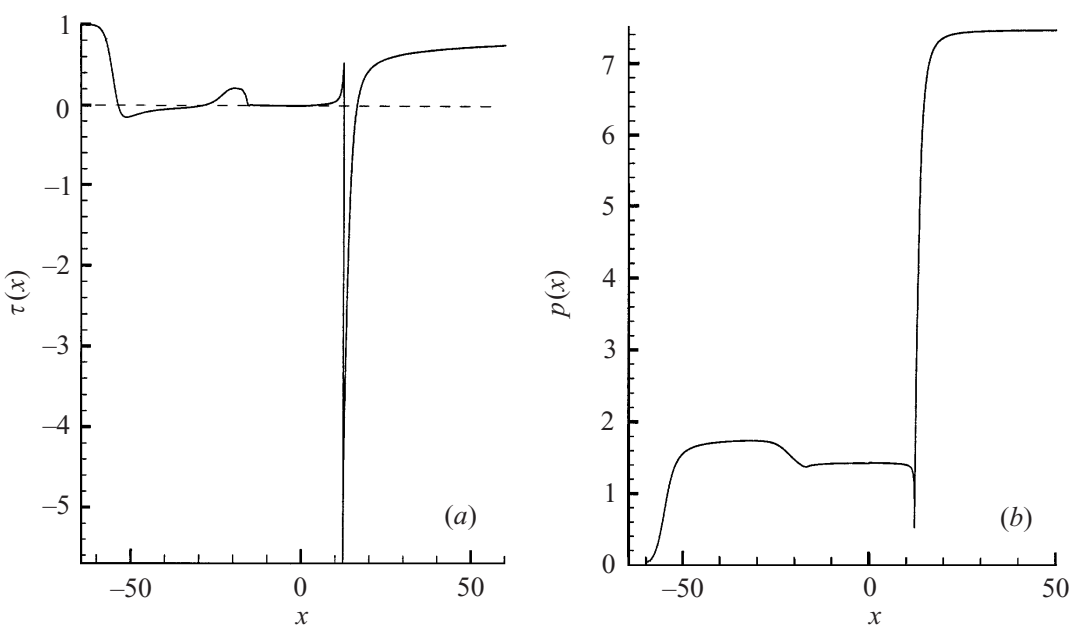

FiguRE 6. (a) Skin friction and ( $b$ ) pressure distributions along the body surface for $\alpha=7.5$. Calculations were performed with method A (mesh $1001 \times 201)$.

then reaches a minimum and starts to rise slowly. While it remains negative, the fluid near the wall is moving in the opposite direction to the rest of the flow. The motion in the reverse flow region is slow. For this reason the pressure develops a 'plateau' which is clearly seen in figure $5(b)$. The ramp angle $\alpha=5$ is large enough for the secondary separation to be observed inside the primary separation region, manifested by a small region of positive skin friction (figure $5 a$ ) and a characteristic drop of the pressure (figure $5 b$ ). Slightly further downstream a sharp second minimum in the skin friction is observed. It is accompanied by a sharp rise in the pressure over the reattachment region. Further downstream the skin friction returns to its unperturbed value $\tau=1$ and the pressure tends to $p=\alpha$ which corresponds to the inviscid flow past the ramp.

For $\alpha$ greater than 5.3, method B started to develop oscillations in the secondary separation region, and we had to rely mainly on method A. The behaviour of the solution for $\alpha=7.5$ is demonstrated in figures 6 and 7. As may be seen from comparing figure 6 with figure 5, near the primary separation point the flow remains unaffected by an increase of $\alpha$. All the fluid dynamic functions including the skin friction $\tau$ and pressure $p$ follow universal laws here as predicted by Neiland (1969) and Stew \& Williams (1969) in their early studies of self-induced separation of the boundary layer on a flat surface. The increase of $\alpha$ merely results in a displacement of the separation point further upstream from the corner $O$.

As can be seen from figure $6(b)$ a second 'plateau' region has been formed in the pressure distribution for $\alpha=7.5$. This is due to the secondary separation which extends from $x=-29.2$ to $x=16.1$, occupying a significant part of the primary separation region (see figure 7). The reattachment of the secondary separation near $x=-29.2$ causes the pressure to rise from one 'plateau' level to another. It is also interesting to observe the formation of the third separation in the streamline pattern for $\alpha=7.5$. The fourth small separation is forming near $x=15.7$ resulting in the inflection of the streamlines clearly visible in figure 7 . Fragmentation of the separation region is expected to continue as $\alpha$ becomes larger.

The accuracy of calculation results has been ensured through various tests which involved different mesh sizes and computational domains. An example of such cal- 


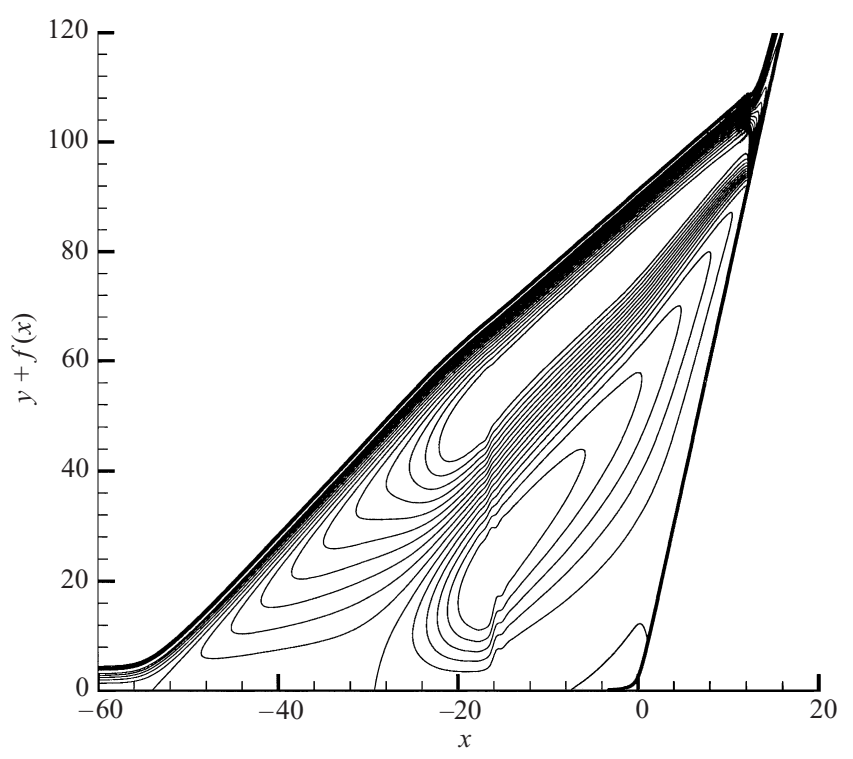

FIGURE 7. The streamline pattern for $\alpha=7.5$.

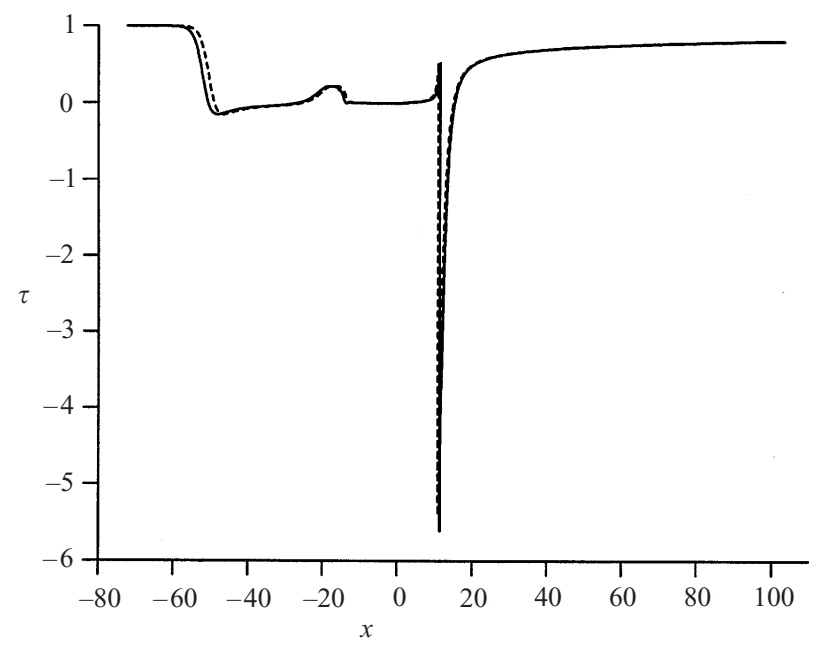

FIGURE 8. Skin friction distribution for $\alpha=7.5$; solid line was calculated with a mesh $1001 \times 201$, dashed line with $701 \times 131$.

culations is presented in figure 8 . It shows that thanks to careful adjustment of mesh density, method A produces reliable results on the mesh $1001 \times 201$ for the ramp angle $\alpha=7.5$. It is also interesting to note that our numerical results prove to be in exceptionally good agreement with the asymptotic formulae

$$
\left.\begin{array}{l}
\tau=1-0.51204 \alpha x^{-2 / 3}+\cdots \\
p=\alpha-0.31763 \alpha x^{-4 / 3}+\cdots
\end{array}\right\} \quad \text { as } \quad x \rightarrow \infty
$$

deduced by Gittler \& Kluwick (1989) for downstream behaviour of the skin friction and pressure (see figure 9). In particular, for the ramp angle $\alpha=7.5$ the numerical solution follows very closely the asymptotic predictions (4.1) for all $x>25$. 

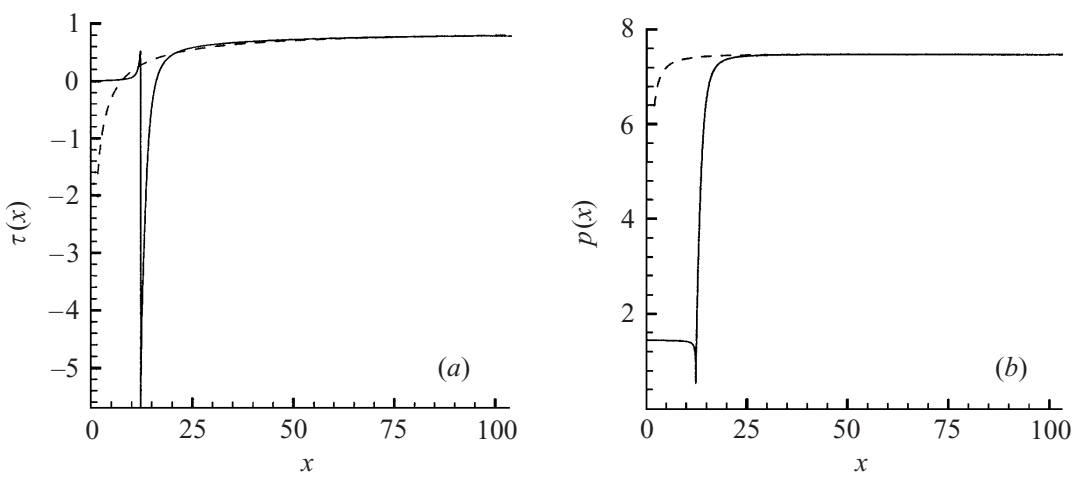

FIGURE 9. Comparison of the numerical solution for $(a)$ skin friction and $(b)$ pressure distribution for $\alpha=7.5$ with asymptotic formulae (4.1); solid line - computation results; dashed line - asymptotic behaviour. Calculations were performed with method A (mesh $1001 \times 201)$.

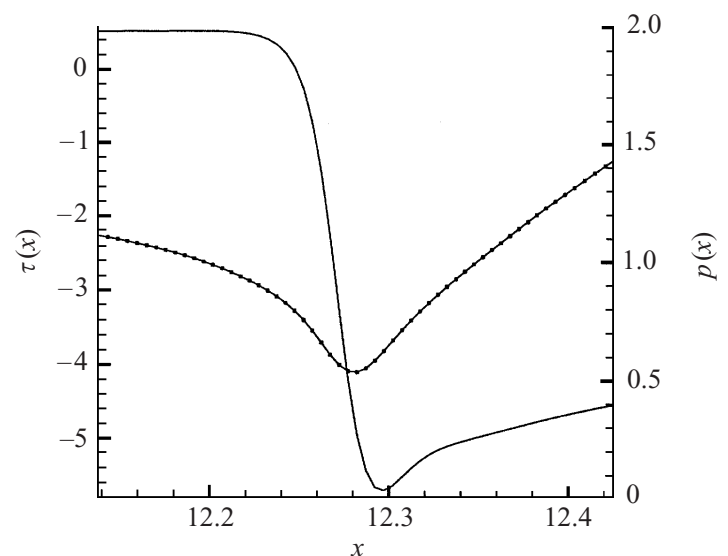

FIGURE 10. Enlargement of skin friction and pressure distributions near the minimum skin friction point for $\alpha=7.5$; circles on the pressure curve show the grid points.

Let us now return to figures $5(a)$ and $6(a)$. We can clearly see that increasing $\alpha$ results in a rapid sharpening of the second minimum in the skin friction $\tau$ prior to the reattachment. This trend was analysed earlier by Smith \& Khorrami (1991). Based on their calculations which (as we will see) over-predicted the growth rate of $\left|\tau_{\min }\right|$, they argued that a singularity develops in the solution at a finite value the compression ramp angle, say $\alpha=\alpha^{*}$, leading to a breakdown of the solution. The singularity was believed to be manifested by unlimited growth of $\left|\tau_{\min }\right|$ as $\alpha \rightarrow \alpha^{*}-0$, and if this were true, it would suggest that the solution does not exist for $\alpha>\alpha^{*}$.

However, our calculations do not support this conclusion. First, we found that the solution remains smooth for all values of $\alpha$ and in our computations there was sufficient resolution to describe the flow behaviour properly despite it becoming more and more complicated as $\alpha$ increases. This is demonstrated by figure 10 which shows enlarged distributions of the skin friction and pressure in a small vicinity of the second minimum for $\alpha=7.5$. Both functions are perfectly smooth, and the circles on the pressure curve show that the method used gives a good resolution of the flow field.

Secondly, the solution follows Neiland's (1970) reattachment theory rather well. Readjusting his arguments for the compression ramp flow (see the Appendix) it may 

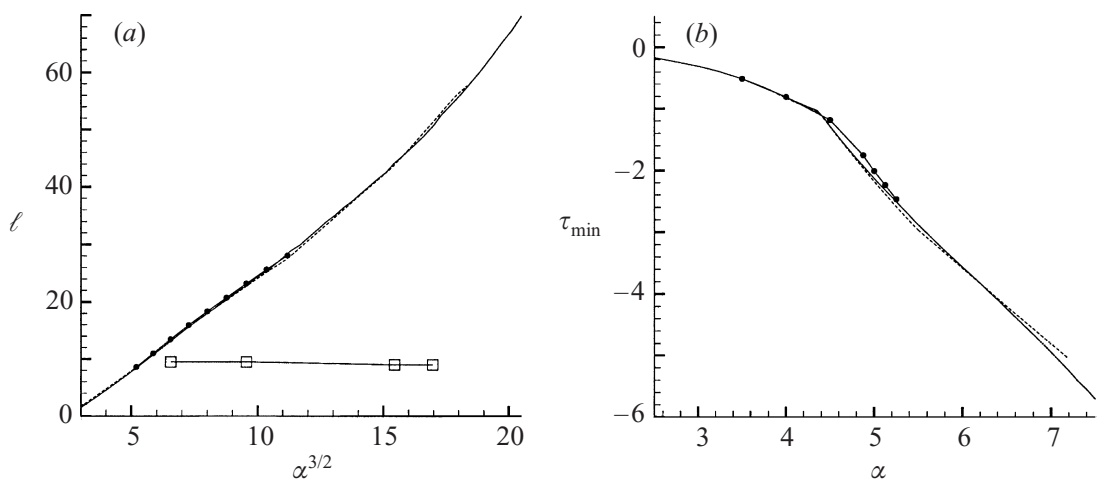

FigURE 11. Confirmation that the calculation results are in good agreement with formulae (4.2) and (4.3) for $(a)$ length of separation region and $(b)$ minimal skin friction; solid line - method A (mesh $1001 \times 201)$; dotted line - method A (mesh $351 \times 101)$; line with circles - method B (mesh $1400 \times 71)$; line with rectangles - Smith $\&$ Khorrami (1991).
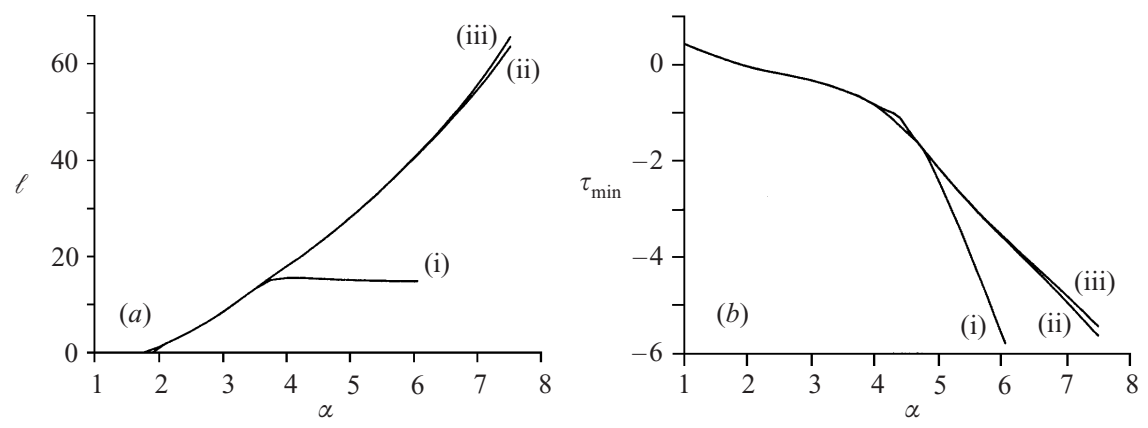

FIGURE 12. Influence of the size of the computational domain on the solution for $(a)$ length of separation region and $(b)$ minimal skin friction; (i) corresponds to $y_{M}=15$, (ii) to $y_{M}=100$ and (iii) to $y_{M}=150$. Calculations were performed using method A (mesh $\left.1001 \times 201 ; \Delta x_{\min }=\Delta y_{\min }=0.005\right)$.

be shown, in particular, that the longitudinal extent of the separation region $\ell$ grows at large values of $\alpha$ as

$$
\ell \sim \alpha^{3 / 2} \text { as } \alpha \rightarrow \infty
$$

while the minimal skin friction

$$
\left|\tau_{\min }\right| \sim \alpha \quad \text { as } \quad \alpha \rightarrow \infty .
$$

Figure 11 reveals that these formulae are in a good agreement with our numerical solution.

To study the effect of the outer boundary we performed calculations with different $y_{M}$. The results are displayed in figure 12 . They clearly show that restricting the outer boundary to, say, $y_{M}=15$ results in significant 'overshooting' in the minimal skin friction. In order to ensure the independence of the numerical results from the size of the computational domain we typically used $y_{M}=120$.

\section{Concluding remarks}

Both numerical methods developed for this study are based on Newton linearization followed by a direct solution of the resulting linear algebraic equations. With this approach the problem of convergence of iterations which was the major concern 
in previous studies does not arise or, to be more precise, is not as acute as with other methods. We found it very advantageous to have two independent methods applied to the same problem. These were used for comparison purposes and for identifying irregularities developing in the solution at large $\alpha$. In particular, we found that the computational domain used by previous authors was not sufficient to resolve the problem. For example, in Ruban's (1978) calculations the upper boundary of the computational domain was placed at $y_{M}=20$, and Smith \& Khorrami (1991) restricted themselves to an even lower value of $y_{M}=10$. The present calculations show that no reliable results can be obtained under these conditions for the compression ramp angle larger than $\alpha=4$.

We further found that an adaptive grid should be used at large values of $\alpha$ when sharp gradients of the sought functions develop in the flow field near the reattachment, the reason being not so much the accuracy of the computational results but rather the convergence of the iteration process. In this respect method A proved to be more flexible than method B. With method B it was difficult to adaptively place points in regions of large gradients. This resulted in the numerical instability encountered with this method at large values of $\alpha$.

Regarding the flow behaviour we found that with increasing $\alpha$ a rapid fragmentation of the separation region takes place with additional eddies forming one inside another. Our calculations did not confirm the finite ramp angle singularity concept put forward by Smith \& Khorrami (1991). The solution does show a sharpening of minimal skin friction prior to reattachment, but this behaviour proved to be well in line with Neiland's (1970) reattachment theory, which does not involve any restrictions on the ramp angle. In fact, it was intended by Neiland for finite values of $\theta$ (see figure 1), i.e. $\alpha \gg 1$.

This observation allows us to give the following conclusion to the discussion of the studies of 'moderate-scale' separations given in the Introduction. In our view, the reason why numerous attempts to develop a rational asymptotic theory of moderatescale separations were not successful does not lie with the finite ramp angle singularity but is a result of oversimplification of the flow. All the theoretical models suggested so far were based on the assumption of single eddy separation which, as we can see, does not represent the real form of the flow.

We gratefully acknowledge the referees for helpful comments and support of the paper. The work presented in this paper was supported by EPSRC through grant GR/L62580/01.

\section{Appendix. Analysis of the reattachment process}

Here the processes taking place near the reattachment point will be discussed following Neiland's (1970) original work. His analysis was devoted to the reattachment of a shear layer which terminates a large-scale separation; correspondingly certain assumptions had been made on the velocity profile across the shear layer as it approaches the wall. Whilst we are dealing here with separation regions that are relatively small, Neiland's description of the process may be easily adjusted for our purposes, as the flow at hand already shows all the essential features of a developed separation. With $\alpha$ becoming large, three major regions are clearly distinguishable in the flow field: the separation region, the main body of the recirculating flow and the reattachment region. We shall start with the separation region. As was mentioned in $\S 5$, the flow behaviour near the separation point $S$ remains independent of the processes taking place downstream. In particular, if we consider the streamline which 


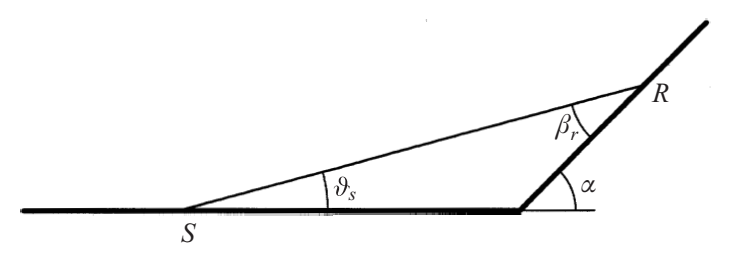

FIGURE 13. A sketch of the 'global' flow structure.

leaves the body surface at the separation point $S$, then the angle made by this streamline with the wall assumes a universal value $\vartheta_{s} \approx 1.6$ as soon as the pressure 'plateau' is reached.

Following this streamline further downstream we see that it should reconnect to the body surface at the reattachment point $R$ for mass conservation to be observed in the recirculation flow region (see figure 13). To predict the shape of this streamline, we can rely on the Ackeret formula $p=\vartheta$ which shows that the streamline slope angle $\vartheta$ remains unchanged as long as the pressure stays constant over the recirculating region. This means that the reattachment angle $\beta_{r}$ may be evaluated as

$$
\beta_{r}=\alpha-\vartheta_{s} \text {. }
$$

With $\alpha$ assumed large, this formula may be approximated by

$$
\beta_{r} \sim \alpha
$$

Note that the assumption of constant pressure in the recirculation region may be relaxed when deriving formula (A 1). Indeed, the variations of pressure from one 'plateau' level to another are small even compared to $\vartheta_{s}$ which has been neglected in (A 1).

Before turning to the reattachment process we need to consider the shear layer developing along the streamline in question which separates the recirculating region from the rest of the flow. Since the fluid in this layer is driven by the constant shear (3.4), the flow is governed by the Neiland's (1971a) self-similar solution (see also Sychev et al. 1998). According to this solution the characteristic thickness of the shear layer grows as the cubic root of the distance from the separation point. Thus prior to the reattachment it is given by

$$
\Delta y \sim \ell^{1 / 3}
$$

where $\ell$ is the distance between the separation and reattachment points. The velocity in the shear layer grows monotonically from the recirculating region towards the outer edge of the layer as shown in figure 14, and its value on the separation streamline may be estimated as

$$
u_{0} \sim \ell^{1 / 3} .
$$

Neiland's (1970) reattachment theory is based on the following concept. As the shear layer impinges on the wall, the inviscid supersonic flow outside the boundary has to turn through angle $\beta_{r}$ to become parallel to the wall downstream of the reattachment point $R$. This causes the pressure to rise by the value

$$
\Delta p=\beta_{r},
$$

and as $\alpha$ increases the pressure gradient induced in the reattachment region starts to dominate the viscous forces. This suggests that the flow in the reattachment region may be treated as inviscid everywhere except, of course, in a thin layer immediately 


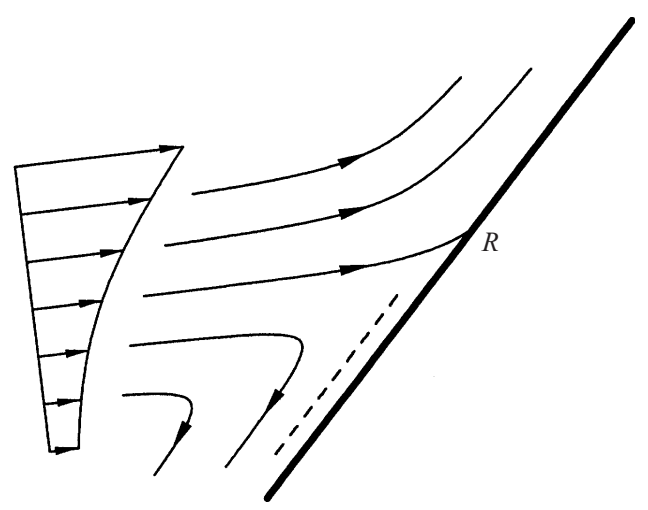

FIGURE 14. The reattachment region.

adjacent to the wall. Taking into account that in any inviscid flow Bernoulli's equation holds, we can write

$$
\frac{1}{2} u^{2}+p=\frac{1}{2}\left[u_{0}(\psi)\right]^{2}+p_{0} .
$$

Here $p_{0}$ is the undisturbed pressure in the recirculation region; the argument $\psi$ in the initial velocity profile $u_{0}(\psi)$ serves as a reminder that Bernoulli's equation should be applied to a particular streamline, all of them taking their origin in the shear layer approaching the reattachment region as shown in figure 14 .

It follows from (A 4) that the streamlines may be subdivided into two categories. In the first one are the streamlines with initial $u_{0}(\psi)$ smaller than some critical value. The fluid particles which travel along these streamlines do not have sufficient kinetic energy to overcome the pressure rise in the reattachment region. If Bernoulli's equation were applied to a streamline from this category assuming $p=p_{0}+\beta_{r}$, then $u^{2}$ would become negative. This means that these fluid particles could overcome only a partial pressure rise in the reattachment region; they cannot pass further downstream but are forced to turn back into the recirculation region.

In the second category are the streamlines with large enough $u_{0}(\psi)$. After passing through the reattachment region they form the boundary layer that originates from the reattachment point and develops along the wall further downstream. The dividing streamline may be identified by applying the condition that $u$ on the left-hand side of equation (A 4) should become zero at the end of the compression process when the streamline comes in contact with the wall. Using this condition we find

$$
\frac{1}{2} u_{0}^{2}=p-p_{0}=\beta_{r} .
$$

For the mass conservation law to hold in the recirculation region the dividing streamline should be the separation streamline. This means that we can use $u_{0}$ as given by (A 3) in formula (A 5). Taking into account that according to (A 1)

$$
\Delta p=p-p_{0} \sim \alpha
$$

we find that the extent of the separation region should grow with $\alpha$ as

$$
\ell \sim \alpha^{3 / 2} .
$$

Formula (A 7) was first deduced by Burggraf (1975) and figure 11(a) reveals an excellent agreement of this formula with our calculation results.

To perform a similar comparison for the minimal skin friction we need to consider a thin viscous sublayer forming near the wall in the reattachment region; it is shown 
in figure 14 by the dashed line. The longitudinal extent $\Delta x$ of the reattachment region may be estimated with the help of the continuity equation (2.4). Comparing the two terms in this equation, we can write

$$
\frac{\partial u}{\partial x} \sim \frac{\partial v}{\partial y} \Rightarrow \frac{u}{\Delta x} \sim \frac{v}{\Delta y} .
$$

In the main inviscid part of the reattachment region $\Delta y$ is defined by (A 2) and the ratio of the velocity components is given by the reattachment angle, i.e. $v / u \sim \beta_{r}$. Hence

$$
\Delta x \sim \alpha^{-1 / 2} .
$$

This estimate is valid for both the inviscid part of the reattachment region and the viscous sublayer. To find the thickness $\delta y$ of the viscous sublayer we turn to the momentum equation (2.3). In the viscous sublayer, by its nature, the viscous term in this equation should be comparable with the pressure gradient

$$
\frac{\partial^{2} u}{\partial y^{2}} \sim \frac{\mathrm{d} p}{\mathrm{~d} x} \Rightarrow \frac{u}{(\delta y)^{2}} \sim \frac{\Delta p}{\Delta x} .
$$

Here $u$ is the characteristic flow velocity in the sublayer. In order of magnitude it coincides with the velocity in the inviscid part of the reattachment region. The latter is given by (A 3). Combining (A 3) with (A 7) we have

$$
u \sim \alpha^{1 / 2} .
$$

Now we can solve (A 9) for $\delta y$ using (A 6) and (A 8). We find

$$
\delta y \sim \alpha^{-1 / 2} .
$$

Using (A 10) and (A 11) it is easy now to evaluate the skin friction

$$
\tau=\frac{\partial u}{\partial y} \sim \frac{u}{\delta y} \sim \alpha
$$

which shows that $\left|\tau_{\min }\right|$ should grow with $\alpha$ as

$$
\left|\tau_{\min }\right| \sim \alpha .
$$

Figure 11(b) shows that our calculation results are in a good agreement with this prediction.

\section{REFERENCES}

BATCHELOR, G. K. 1956a On steady laminar flow with closed streamlines at large Reynolds number. J. Fluid Mech. 1, 177-190.

BATCHELOR, G. K. 1956b A proposal concerning laminar wakes behind bluff bodies at large Reynolds number. J. Fluid Mech. 1, 388-398.

BURGGRAF, O. R. 1975 Asymptotic theory of separation and reattachment of a laminar boundary layer on a compression ramp. In Proc. AGARD Symp. on Flow Separation, Göttingen. AGARDCP-168.

Canuto, C., Hussaini, M. Y., Quarteroni, A. \& Zang, T. A. 1988 Spectral Methods in Fluid Dynamics. Springer.

Chernyshenko, S. I. 1985 On the asymptotics of stationary solutions of the Navier-Stokes equations at large Reynolds numbers. Dokl. Akad. Nauk SSSR 285, 1353-1355.

Chernyshenko, S. I. 1988 The asymptotic form of the stationary separated flow around a body at large Reynolds numbers. Prikl. Mat. Mekh. 52, 746-753. 
GitTleR, P. \& Kluwick, A. 1989 Interacting laminar boundary layers in quasi-two dimensional flow. Fluid Dyn. Res. 5, 29-47.

Goldstein, S. 1948 On laminar boundary-layer flow near a position of separation. Q. J. Mech. Appl. Maths 1, 43-69.

Helmholtz, H. 1868 Über discontinuirliche Flüssigkeits - Bewegungen. Monatsbericht Akad. Wiss. Berlin, pp. 215-228.

IMAI, I. 1953 Discontinuous potential flow as the limiting form of the viscous flow for vanishing viscosity. J. Phys. Soc. Japan 8, 399-402.

ImaI, I. 1957 Theory of bluff bodies. Tech. Rep. BN-104. Inst. Fluid Dyn. and Appl. Math., Univ. Maryland.

KirchHoff, G. 1869 Zur theorie freier Flüssigkeitsstrahlen. J. Reine Angew. Math 70 (4), 289-298.

Landau, L. D. \& Lifshitz, E. M. 1944 Mechanics of Continuous Media. Gostekhizdat, Moscow.

Lighthill, M. J. 1953 On boundary layers and upstream influence. II. Supersonic flows without separation. Proc. R. Soc. Lond. A 217, 478-507.

Messiter, A. F. 1970 Boundary-layer flow near the trailing edge of a flat plate. SIAM J. Appl. Maths 18, 241-257.

NeILAND, V. Y. 1969 Theory of laminar boundary layer separation in supersonic flow. Izv. Akad. Nauk SSSR, Mech. Zhidk. Gaza (4), 53-57.

NeILAND, V. Y. 1970 On asymptotic theory of steady two-dimensional supersonic flows with separation regions. Izv. Akad. Nauk SSSR, Mech. Zhidk. Gaza (3), 22-33.

NeILAND, V. Y. 1971a Flow behind the boundary-layer separation point in a supersonic stream. Izv. Akad. Nauk SSSR, Mech. Zhidk. Gaza (3), 19-25.

NeILAND, V. Y. $1971 b$ On the asymptotic theory of the interaction of supersonic flow with the boundary layer. Izv. Akad. Nauk SSSR, Mech. Zhidk. Gaza (4), 41-47.

Peregrine, D. H. 1985 A note on the steady high-Reynolds-number flow about a circular cylinder. J. Fluid Mech. 157, 493-500.

PrandTL, L. 1904 Über flüssigkeitsbewegung bei sehr kleiner Reibung. In Verh. III. Intern. Math. Kongr., Heidelberg, pp. 484-491. Teubner, Leipzig, 1905.

Rizzetta, D. P., Burggraf, O. R. \& Jenson, R. 1978 Triple-deck solutions for viscous supersonic and hypersonic flow past corners. J. Fluid Mech. 89, 535-552.

Roshko, A. 1967 A review of concepts in separated flow. Proc. Canadian Congr. Appl. Mech. 3, $81-115$.

RUBAN, A. I. 1974 On laminar separation from a corner point on a solid surface. Uch. zap. TsAGI 5 (2), 44-54.

Ruban, A. I. 1978 Numerical solution of the local asymptotic problem of the unsteady separation of laminar boundary layer in supersonic flow. Zh. Vych. Mat. \& Mat. Fiz. 18 (5), 1253-1265.

SADOvSKII, V. S. 1971 Vortex regions in a potential flow with a jump in the Bernoulli constant at the boundary. Prikl. Mat. Mekh. 35 (5), 773-779.

SADOvSKII, V. S. 1973 A study of solutions of the Euler equations containing regions of constant vorticity. Tr. TSAGI (1474).

Sмiтh, F. T. 1985 A structure for laminar flow past a bluff body at high Reynolds number. J. Fluid Mech. 155, 175-191.

Smith, F. T. \& Khorrami, A. F. 1991 The interactive breakdown in supersonic ramp flow. J. Fluid Mech. 224, 197-215.

SQuire, H. B. 1934 On the laminar flow of a viscous fluid with vanishing viscosity. Phil. Mag. 17 (7), 1150-1160.

Stewartson, K. 1969 On the flow near the trailing edge of a flat plate. Mathematika 16, 106-121.

StEWARTSON, K. 1970 On laminar boundary layers near corners. Q. J. Mech. Appl. Maths 23, 137-152.

Stewartson, K. \& Williams, P. G. 1969 Self-induced separation. Proc. R. Soc. Lond. A 312, 181-206.

Sychev, V. V. 1967 On steady laminar flow behind a bluff body at high Reynolds number. In Proc. 8th Symp. on Current Problems of the Mechanics of Liquids and Gases. Tarda, Poland.

SycheV, V. V. 1972 Laminar separation. Izv. Akad. Nauk SSSR, Mech. Zhidk. Gaza (3), 47-59.

Sychev, V. V., Ruban, A. I., Sychev, Vic. V. \& Korolev, G. L. 1998 Asymptotic Theory of Separated Flows. Cambridge University Press.

TAGANOv, G. I. 1970 On the limiting flows of a viscous fluid with steady separation zones as $R e \rightarrow \infty$. Uch. zap. TsAGI 1 (3), 1-14. 